\title{
Intramitochondrial recruitment of endolysosomes mediates Smac degradation and constitutes a novel intrinsic apoptosis antagonizing function of XIAP E3 ligase
}

\author{
A Hamacher-Brady ${ }^{\star, 1,2}$, SC Choe ${ }^{2,3,4}$, J Krijnse-Locker ${ }^{2,5}$ and NR Brady ${ }^{\star 2,3,4}$
}

Intrinsic apoptosis involves BH3-only protein activation of Bax/Bak-mediated mitochondrial outer membrane permeabilization (MOMP). Consequently, cytochrome $c$ is released from the mitochondria to activate caspases, and Smac (second mitochondriaderived activator of caspases) to inhibit XIAP-mediated caspase suppression. Dysfunctional mitochondria can be targeted for lysosomal degradation via autophagy (mitophagy), or directly through mitochondria-derived vesicle transport. However, the extent of autophagy and lysosomal interactions with apoptotic mitochondria remains largely unknown. We describe here a novel pathway of endolysosomal processing of mitochondria, activated in response to canonical BH3-only proteins and mitochondrial depolarization. We report that expression of canonical $\mathrm{BH} 3$-only proteins, $\mathrm{tBid}, \mathrm{Bim}_{\mathrm{EL}}, \mathrm{Bik}, \mathrm{Bad}$, and mitophagy receptor mutants of atypical BH3-only proteins, Bnip3 and Bnip3L/Nix, leads to prominent relocalization of endolysosomes into inner mitochondrial compartments, in a manner independent of mitophagy. As an upstream regulator, we identified the XIAP E3 ligase. In response to mitochondrial depolarization, XIAP actuates Bax-mediated MOMP, even in the absence of BH3-only protein signaling. Subsequently, in an E3 ligase-dependent manner, XIAP rapidly localizes inside all the mitochondria, and XIAP-mediated mitochondrial ubiquitylation catalyses interactions of Rab membrane targeting components Rabex-5 and Rep-1 (RFP-tagged Rab escort protein-1), and Rab5- and Rab7-positive endolysosomes, at and within mitochondrial membrane compartments. While XIAP-mediated MOMP permits delayed cytochrome $c$ release, within the mitochondria XIAP selectively signals lysosome- and proteasome-associated degradation of its inhibitor Smac. These findings suggest a general mechanism to lower the mitochondrial apoptotic potential via intramitochondrial degradation of Smac.

Cell Death and Differentiation (2014) 21, 1862-1876; doi:10.1038/cdd.2014.101; published online 1 August 2014

The intrinsic mitochondrial apoptotic pathway is required for efficient chemotherapeutic killing of cancer cells, ${ }^{1}$ and is initiated through BH3-only protein activation of Bax/Bakmediated mitochondrial outer membrane permeabilization (MOMP). MOMP releases cytochrome $c$ to activate effector caspases. ${ }^{2}$ Conversely, inhibitor of apoptosis protein (IAP) family members suppress initiator and effector caspases via direct binding and E3 ligase activities. ${ }^{3-5}$ Consequently, MOMP-induced release of Smac (second mitochondriaderived activator of caspases) from the mitochondria, to inhibit XIAP (X-chromosome-linked IAP)-mediated caspase suppression, can be required for apoptosis. ${ }^{6}$

Autophagy, a lysosomal degradative mechanism undergoing extensive crosstalk with cell death and survival pathways, ${ }^{7}$ degrades damaged mitochondria in a process termed mitophagy. ${ }^{8,9}$ Damaged mitochondria are targeted by lysosomal degradation through the recruitment of autophagy receptors to the outer mitochondrial membrane $(\mathrm{OMM}),{ }^{8}$ or via delivery of mitochondrial-derived vesicles (MDVs) directly to the lysosome. ${ }^{10}$ The E3 ubiquitin ligase Parkin targets and ubiquitylates mitochondria, mediating both MDV degradation $^{11}$ and autophagy receptor-dependent mitophagy. ${ }^{12,13}$ Alternatively, Fundc $1^{14}$ and atypical $\mathrm{BH} 3-$ only Nip family proteins Bnip3 and Bnip3L/Nix localize to the OMM and act as mitophagy receptors via their LC3-interacting region (LIR). ${ }^{15-18}$ While targeting of damaged mitochondria suggests that mitophagy may counter apoptotic mitochondria, mitophagy occurs progressively over days, ${ }^{12,14,16,17,19}$ a rate that is likely insufficient to alter intracellular propagation of mitochondrial apoptosis, which can occur within minutes. ${ }^{20,21}$ Indeed, Bnip3- and Fundc1-induced mitophagy have no direct effect on apoptosis, ${ }^{14,18}$ and we determined that Bnip3-mediated mitophagy was cytoprotective if activated before apoptosis. ${ }^{17}$ While MDV delivery of mitochondria to lysosomes operates at

\footnotetext{
${ }^{1}$ Lysosomal Systems Biology, German Cancer Research Center (DKFZ), Heidelberg, Germany; ${ }^{2}$ Bioquant, University of Heidelberg, Heidelberg, Germany; ${ }^{3}$ Systems Biology of Cell Death Mechanisms, German Cancer Research Center (DKFZ), Heidelberg, Germany; ${ }^{4}$ Department of Surgery, Heidelberg University Hospital, Heidelberg, Germany and ${ }^{5}$ Department of Virology, Hygiene Institute, University of Heidelberg Medical School, Heidelberg, Germany

*Corresponding authors: A Hamacher-Brady, Lysosomal Systems Biology, German Cancer Research Center (DKFZ), Im Neuenheimer Feld 267, BQ0045, Heidelberg 69120, Germany. Tel: +49 6221 5451234; Fax: +49 6221 5451482; E-mail: a.brady@dkfz.de

or NR Brady, Systems Biology of Cell Death Mechanisms, German Cancer Research Center (DKFZ) and Heidelberg University Hospital, Im Neuenheimer Feld 267, BQ0037, Heidelberg 69120, Germany. Tel: +49 6221 5451234; Fax: +496221 5451482; E-mail: n.brady@dkfz.de

Abbreviations: BafA1, bafilomycin A1; CCCP, carbonyl cyanide $m$-chlorophenyl hydrazine; FRAP, fluorescence recovery after photobleaching; GFP, green fluorescent protein; IAP, inhibitor of apoptosis; IF, immunofluorescence; LIR, LC3-interacting region; MIU, motif interacting with ubiquitin; MDVs, mitochondrial-derived vesicles; MOMP, mitochondrial outer membrane permeabilization; OMM, outer mitochondrial membrane; RING, really interesting new gene; RFP, red fluorescent protein; $\mathrm{ROI}$, region of interest; Smac, second mitochondria-derived activator of caspases; XIAP, X-chromosome-linked IAP

Received 12.7.13; revised 21.5.14; accepted 30.5.14; Edited by JM Hardwick; published online 01.8.14
} 
a higher rate, minutes to hours, ${ }^{10}$ this process is regulated by Parkin and restricted to specific mitochondrial components. ${ }^{11}$ Overall, for most intrinsic apoptosis scenarios it remains unknown whether lysosomal processing of mitochondria influences their capacity to activate or enhance apoptosis.

Here, we used high-resolution imaging to evaluate the behavior of apoptosis, autophagy, lysosomal and ubiquitylation pathways in response to canonical (tBid, Bim $\mathrm{EL}_{\mathrm{L}}, \mathrm{Bik}, \mathrm{Bad}$ ) and atypical (Bnip3, Bnip3L/Nix) BH3-only protein expression. We report that, in parallel to intrinsic apoptosis signaling, canonical $\mathrm{BH} 3-$ only proteins induce the recruitment of endolysosomal machinery, in the absence of mitophagy. We determined that mitochondrial depolarization rapidly translocates the caspase inhibitor XIAP to the mitochondria. There, XIAP actuates MOMP within all mitochondria, concomitant with ubiquitylation at the OMM and inside OMM-bound regions, and triggers ubiquitin-dependent recruitment of Rab5 and its binding partners, as well as late endosomes into the mitochondria. Consequently, in a manner dependent on lysosome- and proteasome-activities, XIAP degrades its inhibitor Smac. We propose that in response to bioenergetic stress, the functional integration between lysosomes and mitochondria, mediated by XIAP and independent of autophagy, offers a novel mechanism to modulate mitochondrial apoptosis.

\section{Results}

Canonical BH3-only proteins induce autophagyindependent endolysosomal entry into the mitochondria. To activate specific mitochondrial apoptosis programs, we expressed fluorescent protein-tagged canonical activator (tBid, Bim $\mathrm{EL}_{\mathrm{L}}$ ) and inhibitor (Bad, Bik), ${ }^{22,23}$ and atypical (Bnip3 and $\mathrm{Nix})^{24} \mathrm{BH} 3$-only proteins. To elucidate events upstream and downstream of MOMP, we used caspase-3-deficient MCF7 breast cancer cells ${ }^{25}$ lacking caspases 3-8 feedback amplification of apoptosis ${ }^{26}$ and inhibition of autophagy. ${ }^{27}$ To assess mitophagy induction, we investigated the colocalization of mitochondria with autophagy marker Atg8 proteins LC3B and GATE16. ${ }^{28}$ As expected, at $48 \mathrm{~h}$ of expression mitochondria targeted by the atypical $\mathrm{BH} 3-$ only proteins, red fluorescent protein (RFP)-Bnip3 and RFP-Nix, which contain an LIR mediating Atg8-dependent mitophagy, ${ }^{16,17}$ were positive for GFP-LC3B and GFP-GATE16, respectively (Figure 1a). In contrast, canonical tBid-RFP and RFP-Bim ${ }_{E L}$, examined at $24 \mathrm{~h}$ of expression owing to higher toxicity, did not induce mitochondrial sequestration by autophagosomes (Figure 1a).

Next, we examined the subcellular localization of the GTPase Rab7, an endolysosomal marker. ${ }^{29}$ Mito-RFPlabeled control mitochondria did not colocalize with GFP-Rab7 (Figure 1b). Surprisingly, in response to $24 \mathrm{~h}$ expression of tBid-RFP and RFP-Bim $E$, GFP-Rab7 colocalized with the Tom20-immunolabeled mitochondrial compartment. At this time-point, wild-type (WT) RFP-Nix-targeted mitochondria did not colocalize with GFP-Rab7 (Figure 1b), consistent with the slow induction of mitophagy by its homolog Bnip3. ${ }^{17}$ However, Nix deleted for its LIR (RFP-Nix $\Delta$ LIR) induced GFP-Rab7 accumulation with mitochondria at $24 \mathrm{~h}$. Similar results were obtained for canonical RFP-Bad and RFP-Bik, and RFP-Bnip3 versus RFP-Bnip3 $\Delta$ LIR (Figure 1b).
Remarkably, high-resolution imaging suggested that mitochondria targeted by canonical or LIR-mutated atypical $\mathrm{BH}$ only proteins contained GFP-Rab7-positive vesicles inside OMM-bound regions (regions of interest (ROIs) of Figures $1 \mathrm{~b}$ and d; plot profile in Supplementary Figure S3a), unlike during mitophagy where autophagolysosomes are found surrounding mitochondria. To maximize axial resolution, Z-stacks acquired with $0.15 \mu \mathrm{m}$ step sizes were acquired. Importantly, tBid-RFP fully localized to Tom20-labeled mitochondria (Supplementary Figures $\mathrm{S} 1 \mathrm{a}$ and $\mathrm{b}$ ). Within the threedimensional (3D) volume of a single tBid-targeted mitochondrion, a $180^{\circ}$ axial slice rotation of tBid-RFP, GFP-Rab7 and mitochondrial matrix-localized TRAP1 indicates the intramitochondrial concentration of GFP-Rab7 (Supplementary Figures S1c and d).

Intramitochondrial localization of GFP-Rab7 vesicles within tBid-targeted mitochondria was further supported by profile analysis of N-SIM super-resolution microscopy images (Supplementary Figure S2). Moreover, electron microscopy demonstrated that RFP-Nix $\Delta$ LIR- and tBid-RFP-targeted mitochondria lacked the typical double-membraned engulfment observed with autophagy (Figure 1c). Instead, those $\mathrm{BH} 3-$ only protein-targeted mitochondria displayed discontinuities of the OMM (open arrows), and contained electrondense vesicles (asterisks). Mitochondrial identity of these aberrant structures was verified by immunogold labeling of OMM protein Tom20. Detection of immunogold-labeled GFPRab7 (closed arrows) inside the OMM further supported mitochondrial entry of Rab7.

Quantification of the subcellular fluorescence distribution revealed that approximately $50 \%$ of cellular GFP-Rab7

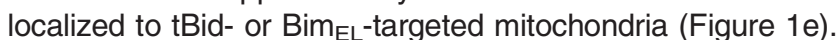
Importantly, immunofluorescence (IF) staining demonstrated the accumulation of endogenous Rab7 at tBid-RFP- and RFP-Bim $\mathrm{EL}_{\mathrm{L}}$-targeted mitochondrial compartments, confirming pathway activation in the absence of Rab7 overexpression (Figure $1 \mathrm{f}$ and Supplementary Figure S3a).

Taken together, the above findings suggest a pathway activating autophagy-independent endolysosomal vesicle entry into the mitochondria during canonical BH3-only protein-mediated mitochondrial apoptosis.

Canonical BH3-only proteins induce intramitochondrial endosomal trafficking. To determine the source and function of this pathway, we further investigated Rab GTPase regulation of membrane trafficking. Rab5 coordinates with Rab7 to regulate endosomal maturation. ${ }^{30}$ In response to tBid-RFP expression, endogenous Rab5 concentrated inside the Tom20-immunolabeled mitochondrial compartment (Figure 2a and Supplementary Figure S3a). Similarly, GFPfused tBid, Bim $E_{E L}$, Bad, Bik, Nix $\Delta$ LIR and Bnip3 $\Delta$ LIR, but not Bnip3 and Nix, induced the mitochondrial accumulation of RFP-Rab5 (Figure 2b). Approximately $70 \%$ of cellular Rab5 translocated from the cytosol to tBid-GFP- and GFP-Bim $\mathrm{EL}^{-}$ targeted mitochondria (Figure $2 \mathrm{c}$ ). The $\mathrm{BH} 3$-domain-inactive mutant tBid(G94E)-RFP ${ }^{31}$ did not induce mitochondrial GFPRab5 recruitment (Figure $2 d$ ). These findings suggest that mitochondrial recruitment of Rab5 is a general response to a canonical proapoptotic $\mathrm{BH} 3$-only protein stimulus, and occurs in the absence of LIR-mediated mitophagy. 
a

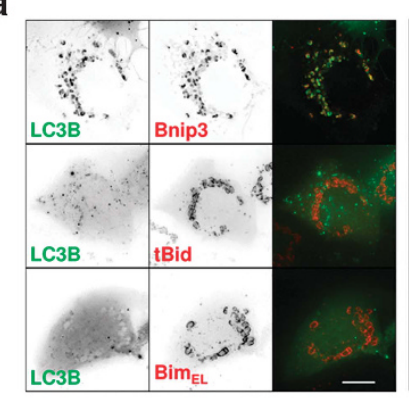

c

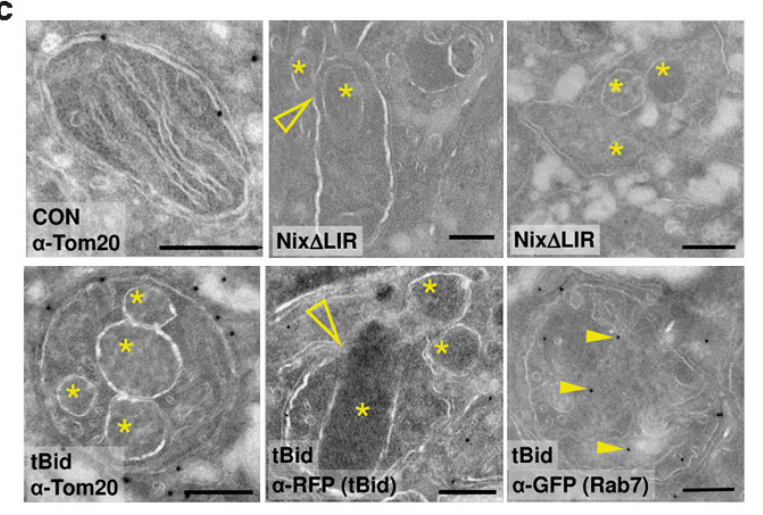

d

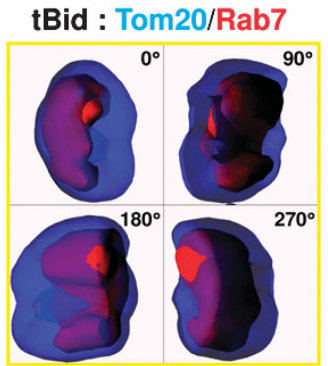

e

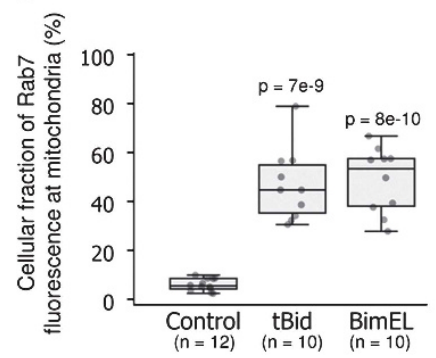

b

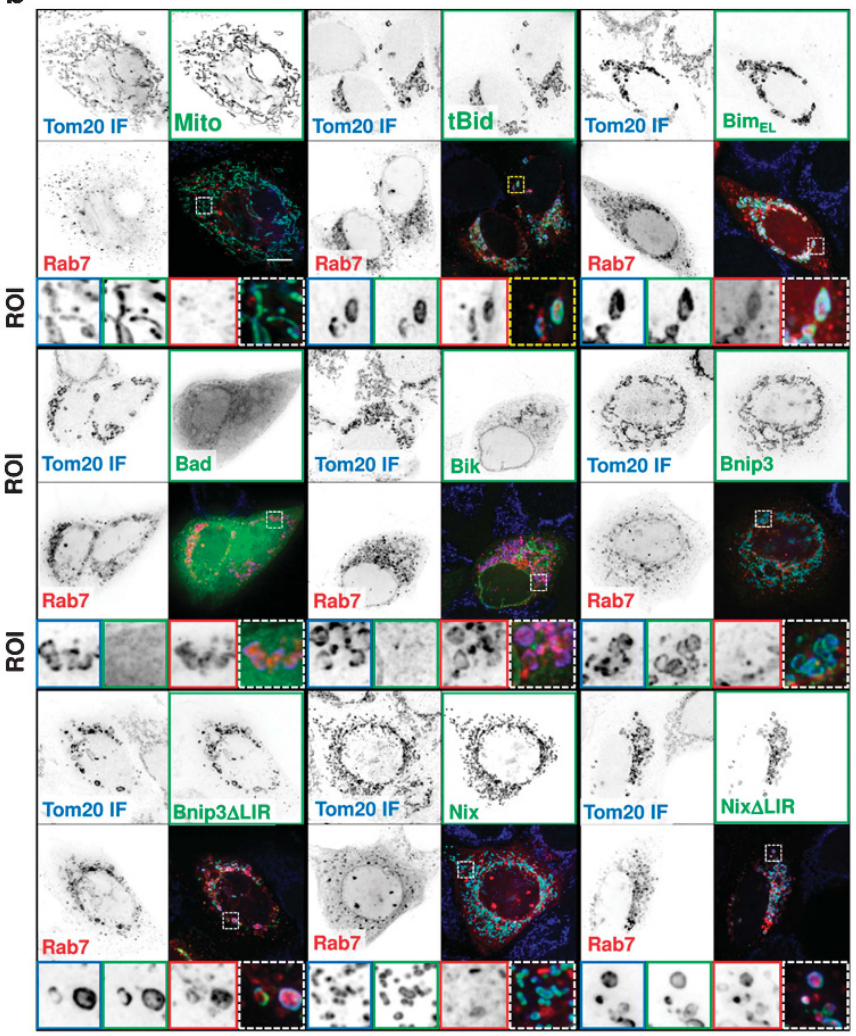

f

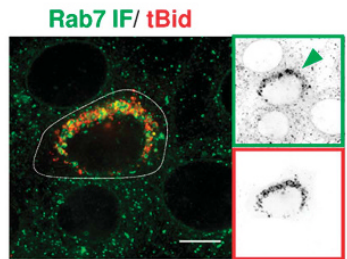

$\operatorname{Rab7}$ IF/ Bim $\mathrm{EL}$

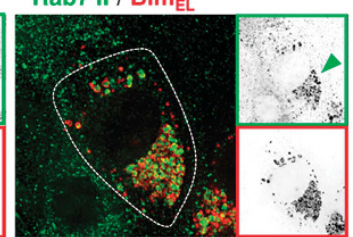

Figure 1 Canonical BH3-only proteins induce intramitochondrial entry of Rab7-positive endolysosomes, in the absence of mitophagy. (a) MCF7 cells coexpressing GFP-LC3 or GFP-GATE16 with RFP-tagged BH3-only proteins tBid and Bim EL $_{2}(24 \mathrm{~h})$, or Nix and Bnip3 (48 h). Scale bar, $10 \mu \mathrm{m}$. (b) GFP-Rab7 coexpression with mito-RFP or RFP-tagged BH3-only proteins tBid, BimeL, Bad, Bik, Bnip3, Bnip3 $\Delta$ LIR, Nix or Nix $\Delta$ LIR (24 h). IF detection of OMM marker Tom20. Scale bar, $10 \mu \mathrm{m} ; \mathrm{ROI}, 5.4 \times 5.4 \mu \mathrm{m}^{2}$. (c) Electron micrographs of mito-RFP (Con)- and tBid-RFP-targeted mitochondria $(24 \mathrm{~h})$ immunogold labeled with $\alpha$-Tom20 or $\alpha$-RFP for tBid-RFP as indicated, Nix $\Delta$ LIRtargeted mitochondria (48 h), and tBid-RFP-targeted mitochondria ( $24 \mathrm{~h}$ ) immunogold labeled with $\alpha$-GFP for GFP-Rab7. Scale bars, $200 \mathrm{~nm}$. (d) 3D surface renderings of RFP-Rab7- (red) and Tom20-immunolabeled OMM (blue) of a tBid-targeted mitochondrion (see (b), yellow box). (e) Box plots represent the cellular fraction of GFP-Rab7 fluorescence colocalizing with Tom20-labeled mitochondria. ' $n$ ' signifies the number of representative cells analyzed. $P$-values describe statistical significance versus control (Student's t-test). (f) IF of Rab7 in MCF7 cells expressing tBid-RFP or RFP-Bim $\mathrm{EL}_{\mathrm{L}}(24 \mathrm{~h})$

Next, we explored the dynamics and mechanisms of endosomal recruitment to mitochondria. FRAP (fluorescence recovery after photobleaching) applied to RFP-tBid-targeted mitochondria revealed that GFP-Rab5 recovered in $<1$ min following bleaching (Figures $3 a$ and $b$ ), a rate slightly greater than non-mitochondrial GFP-Rab5-positive endosomes in control cells expressing mito-RFP (Figure 3b). Within the same time period, bleached tBid-RFP inside the ROI did not recover (Figure 3a), demonstrating that GFP-Rab5 recovery was due to active endosomal membrane trafficking rather than mitochondrial fusion. FRAP of GFP-Rab7 localizing to tBid-targeted mitochondria revealed a comparable, rapid recovery, slightly slower than GFP-Rab5 (Figures 3b and c). Recoveries of Rab5 and Rab7 are consistent with previously reported recovery rates for early and late endosomes. ${ }^{32,33}$
Further, unlike WT GFP-Rab7, dominant-inactive GDP-bound GFP-Rab7(T22N) ${ }^{29}$ did not localize to tBid-RFP-targeted mitochondria (Figure 3d). Thus, endosomal membrane trafficking is actively engaged in mitochondria stressed by canonical BH3-only proteins.

As a targeting mechanism to direct trafficking to mitochondria, we investigated Rabex-5 (Rab5 guanine nucleotide exchange factor), ${ }^{34}$ which can determine intracellular localization of Rab5 to specific membranes. ${ }^{35}$ In response to tBid-RFP expression, endogenous Rabex-5 concentrated at mitochondria (Figures $3 e$ and d), and 3D rendering of individual mitochondria suggested its presence at the OMM and within inner mitochondrial compartments (yellow arrow). Also RFP-Rabex-5 appeared to localize at the OMM and inside OMM-bound regions of cells with 
a

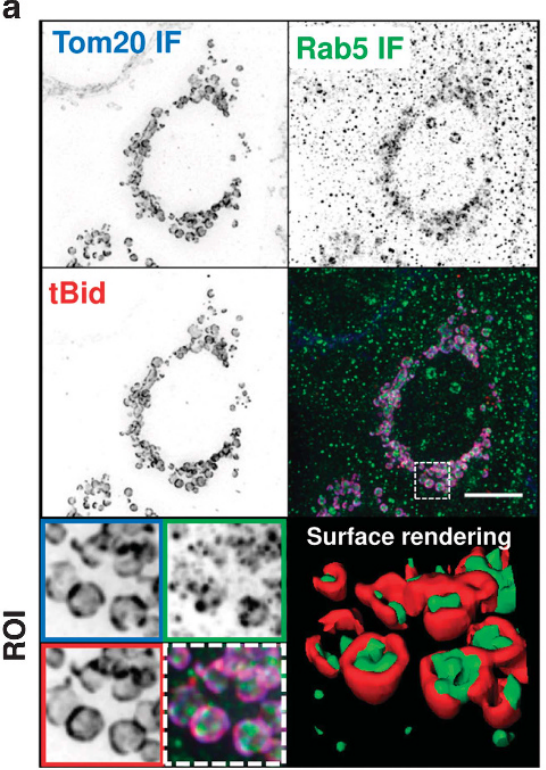

C

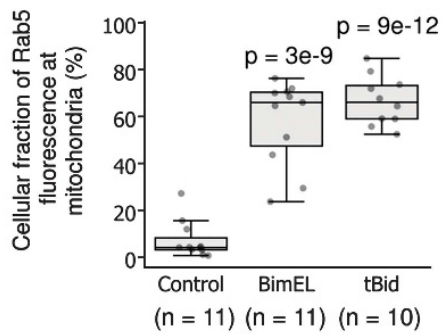

d

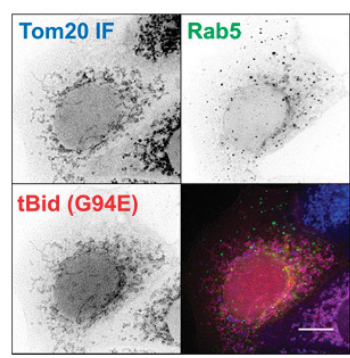

b

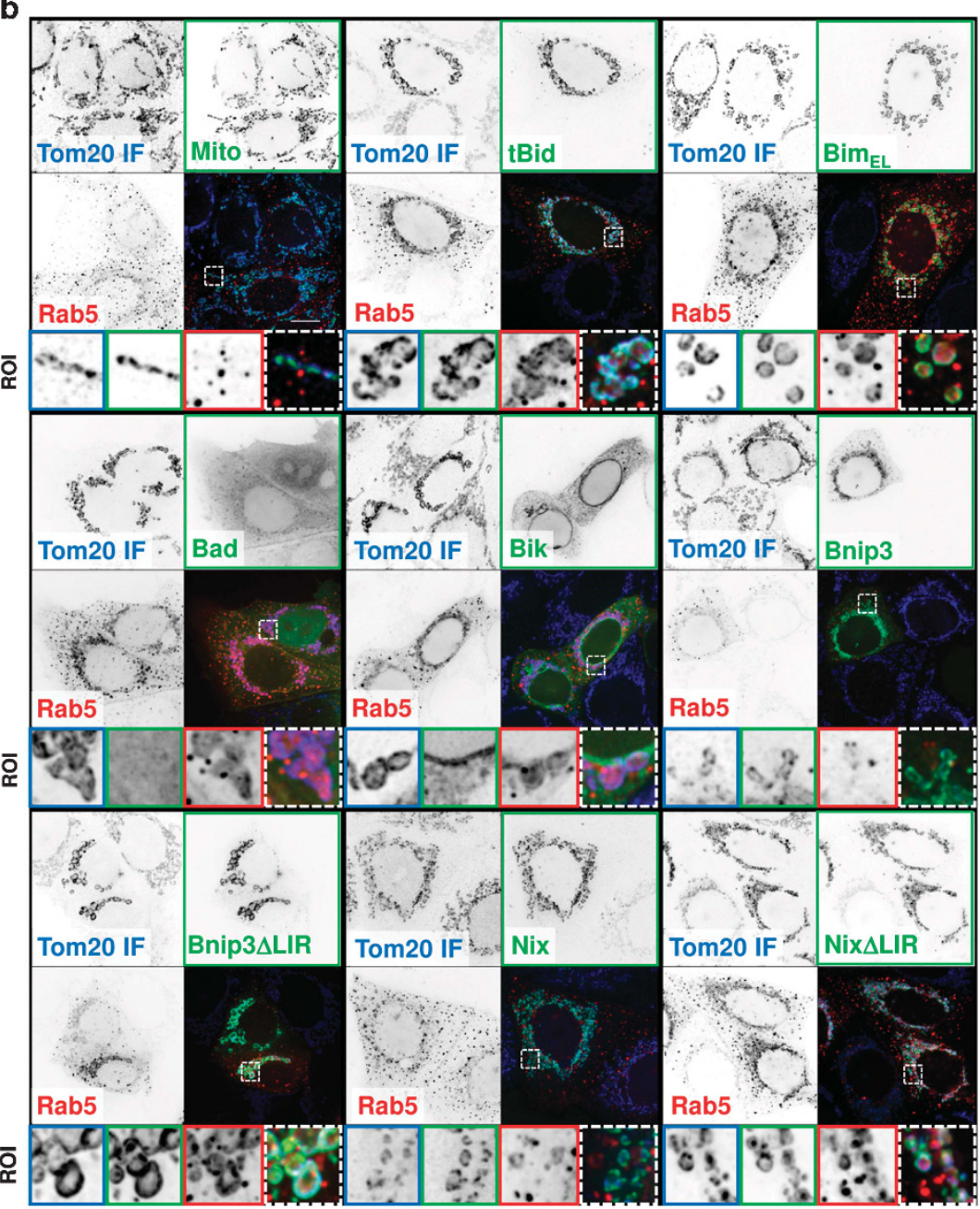

Figure 2 Early endosomal vesicle entry into canonical BH3-only protein- and LIR-mutated atypical BH3-only protein-targeted mitochondria. (a) IF of OMM protein Tom20 and Rab5 in tBid-RFP-expressing cells (24 h). 3D surface rendering of Rab5- and tBid-RFP-targeted mitochondria within ROI. (b) GFP-Rab5 coexpression with mito-RFP or

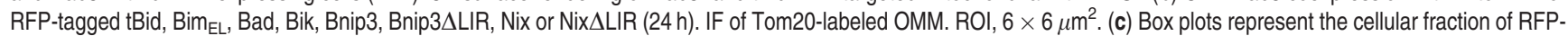
Rab5 fluorescence colocalizing with Tom20-immunolabeled mitochondria. ' $n$ ' signifies the number of cells analysed. $P$-values describe statistical significance versus control (Student's t-test). (d) BH3-inactive mutant tBid(G94E)-RFP coexpression with GFP-Rab5 (24h). IF of Tom20

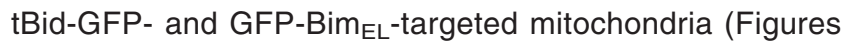
$3 f$ and $g$ ). Notably, $3 \mathrm{D}$ rendering suggested that RFP-Rabex-5 and tBid-GFP were restricted to separate domains on the OMM (Figures $3 e$ and $f$ ), implying a biochemical transformation of the OMM.

As Rabex-5 can direct endosomal trafficking to specific cellular compartments, ${ }^{35}$ we asked how its localization to mitochondria was regulated. Ubiquitylation regulates endosomal trafficking ${ }^{36}$ and targets proteins and organelles for lysosomal degradation. ${ }^{8,13,37}$ Rabex-5 contains a motif interacting with ubiquitin (MIU), which determines its recruitment. $^{38}$ RFP-Rabex-5(A58G), with a mutated MIU domain resulting in reduced ubiquitin binding, ${ }^{39}$ did not localize to

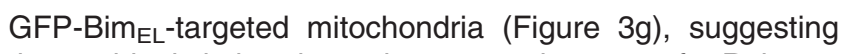
that ubiquitylation-dependent recruitment of Rabex-5 regulates endosomal targeting of mitochondria.

\section{Mitochondria targeted by canonical BH3-only proteins} are ubiquitylated at the OMM and inside OMM-bound regions. To further test pathway regulation by ubiquitylation, we examined whether mitochondria are ubiquitylated in response to canonical $\mathrm{BH} 3-$ only protein expression. Indeed, in RFP-Bim EL-expressing cells, GFP-ubiquitin $^{40}$ (GFP-Ub) colocalized with the circular Tom20-labeled OMM (Figure 4a, solid arrow; Supplementary Figure S3a). In addition, punctate GFP-Ub fluorescence was found inside OMM-bound 
regions (Figure 4a, open arrow). To investigate the nature of ubiquitylation events, we tested for lysine 63 (K63) polyubiquitylation using the K63 linkage-specific sensor Vx3K0-
GFP. ${ }^{41}$ tBid-RFP expression induced Vx3K0-GFP cluster formation inside Tom20-bound regions (Figure 4b), but not at the OMM as detected with GFP-Ub. The BH3 mutant
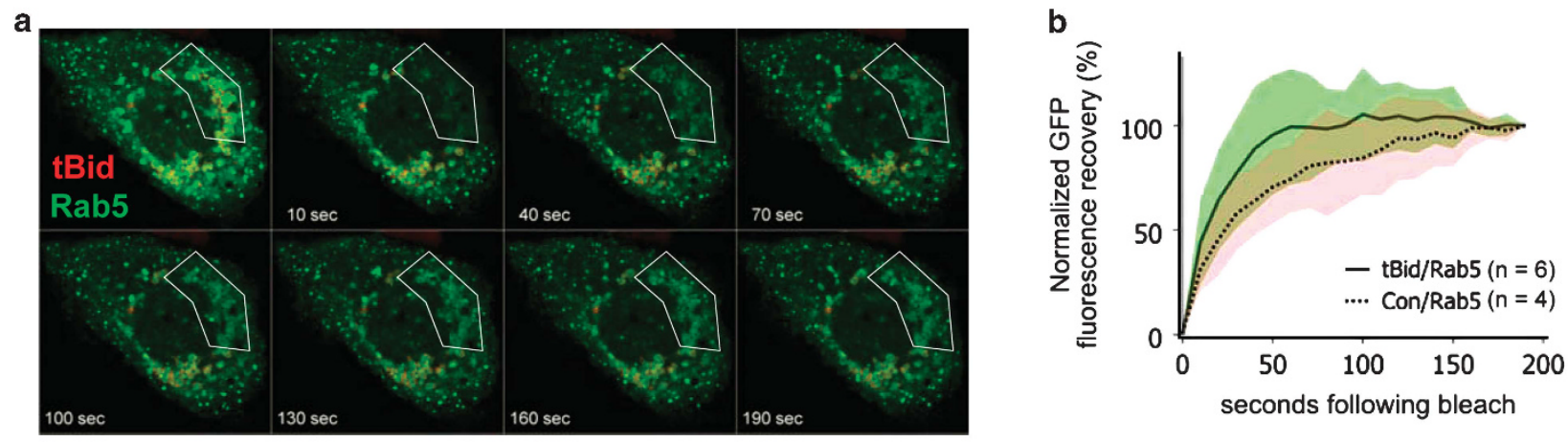

c
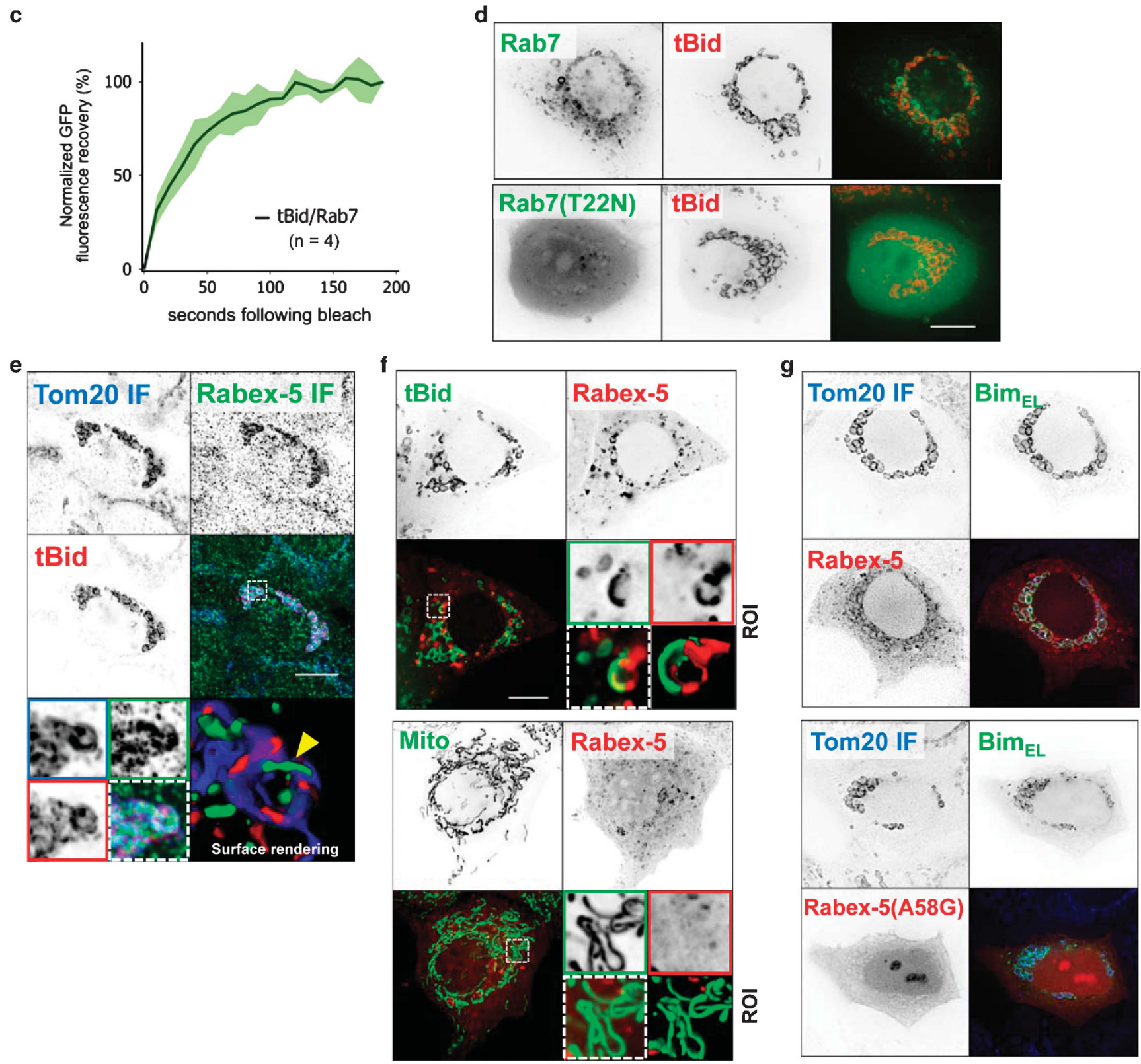
tBid(G94E)-RFP, which does not bind Bcl-2 and Bax, ${ }^{31}$ neither activated endolysosomal colocalization with mitochondria (Figure 2d) nor induced mitochondrial Vx3K0-GFP localization (Figure 4b). Moreover, K63 chains were absent from RFP-Nix-targeted mitochondria, but were colocalizing with RFP-Nix $\Delta$ LIR-targeted mitochondria (Figure 4c). Consistent with Vx3K0-GFP findings, tBid-RFP- and RFPBim $_{\mathrm{EL}}$-targeted mitochondria exhibited prominent immunolabeling of $\mathrm{K} 63$ chain clusters inside OMM-bound regions (Figure 4d). Immunolabeled K48 chains were similarly detected as clusters inside OMM-bound regions (Figure $4 \mathrm{e}$ ). Taken together, these findings suggest that canonical $\mathrm{BH} 3-$ only proteins induce an initial OMM ubiquitylation (non-K63/K48), followed by intramitochondrial K63 and K48 chaining, thus implicating pathway regulation by an apoptosis-associated ubiquitin ligase.

Intramitochondrial accumulation of endolysosomes is dependent on XIAP E3 ligase and triggered by mitochondrial depolarization. IAP family members can inhibit caspase activities by binding via baculovirus AIP repeat (BIR) domains, and through ubiquitin ligase activity. ${ }^{3-5}$ The most potent member, XIAP, ${ }^{4,42}$ contains a RING (really interesting new gene) domain with E3 ligase activity required for antiapoptotic activity. ${ }^{43}$ As XIAP was suggested to localize to mitochondria during apoptosis, ${ }^{44}$ we examined its localization in response to $\mathrm{BH} 3-0 n l y$ protein targeting of the mitochondria. RFP-Bim $\mathrm{EL}_{\text {L }}$ expression induced translocation of endogenous XIAP (Figure 5a) and GFP-XIAP (Figure 5b) to the mitochondria, whereas in non-transfected cells XIAP remained diffuse in the cytosol.

As mitochondria become bioenergetically compromised as a major consequence of MOMP, ${ }^{45}$ we asked whether XIAP translocation directly necessitates $\mathrm{BH}$ domain signaling, or rather, is a response to mitochondrial energetic stress. Therefore, we induced mitochondrial depolarization with the protonophore carbonyl cyanide m-chlorophenyl hydrazine (CCCP) in combination with XIAP overexpression. Following $3 \mathrm{~h}$ CCCP $(20 \mu \mathrm{M})$ treatment, in cells expressing RFP-XIAP, endogenous Rab5 and RFP-XIAP colocalized with mitochondria (Figure $5 \mathrm{c}$ ). Quantifications revealed that $\sim 10 \%$ of cellular RFP-XIAP translocated to the mitochondria (Figure 5d). In cell populations, before treatment $\sim 20 \%$ of RFP-XIAP-expressing cells exhibited mitochondrial $\mathrm{XIAP}$, increasing to $\sim 80 \%$ of cells in response to CCCP (Figure $5 e$ ). Furthermore, $\sim 25 \%$ of total endogenous Rab5 (Figure 5f) localized adjacent to RFP-XIAP at the OMM and inside OMM-bound regions (Figure 5c, 3D surface rendering; Supplementary Figure S3b).
To further causatively link XIAP pathway activity with the mitochondrial recruitment of Rab5, we generated cell lines with shRNAs targeting XIAP. Approximately $30 \%$ of control shRNA cells displayed CCCP-induced mitochondrial RFPRab5. This higher response, compared with transient expression experiments, might originate from the use of puromycin for stable cell line maintenance. Importantly, both XIAP knockdown lines exhibited significantly decreased Rab5 entry into depolarized mitochondria (Figure $5 \mathrm{~g}$ ).

XIAP E3 ligase activity is responsible for the ubiquitylation of depolarized mitochondria. As Rab5 translocation to depolarized mitochondria was XIAP-dependent, we asked if XIAP activates ubiquitylation of depolarized mitochondria. In response to CCCP, $\mathrm{V} \times 3 \mathrm{~K} 0-\mathrm{GFP}$ reported prominent $\mathrm{K} 63$ chaining specifically at RFP-XIAP-targeted mitochondria (Figure 6a). Immunolabeling confirmed mitochondrial K63 chaining, and further evidenced prominent K48 chaining at RFP-XIAP-containing mitochondria (Figure 6b). Moreover, endogenous Rabex-5 and RFP-Rabex-5 localized to depolarized mitochondria, whereas ubiquitin binding-deficient RFPRabex-5(A58G) showed no translocation (Figures 6c and d). This suggests that XIAP ubiquitylates mitochondria upstream of endosomal entry. Additionally evidencing recruitment of endosomal trafficking machineries, RFP-tagged Rab escort protein-1 (Rep-1), which binds and transports Rab5 and Rab7, ${ }^{46}$ and endogenous Rab7 were recruited to the mitochondria of XIAPexpressing, CCCP-treated cells (Figures $6 \mathrm{e}$ and f).

XIAP action is distinct from Parkin-mediated mitophagy. Mitochondrial depolarization can activate the E3 ligase Parkin, which mediates mitophagy through ubiquitylation of OMM proteins and recruitment of autophagy receptors. ${ }^{12,13,47}$ Thus, we directly compared XIAP action at mitochondria with Parkin-mediated mitophagy. Similar to our results with canonical $\mathrm{BH} 3-$ only proteins (Figure 1), but unlike what has been reported for Parkin, ${ }^{12}$ CCCP treatment did not induce mitophagy in RFP-XIAP-expressing cells (Supplementary Figure S1a). Conversely, RFP-Parkin overexpression did not induce mitochondrial recruitment of GFPRab5 in response to CCCP (Supplementary Figure S1b). Moreover, CCCP in combination with RFP-Parkin expression resulted in $\mathrm{K} 63$ chaining at the $\mathrm{OMM}$, as indicated by colocalization of Vx3K0-GFP with the circular Tom20-labeled OMM (Supplementary Figure S4c), whereas CCCP-induced K63 chaining was observed as punctate foci inside OMMbound regions in RFP-XIAP-expressing cells (Figures 6a and b). Importantly, after $3 \mathrm{~h}$ of CCCP treatment, GFP-XIAP targeted Rab7 to the entire mitochondrial population

Figure 3 Active recruitment of endolysosomes to canonical BH3-only protein-targeted mitochondria. (a-c) FRAP analysis of GFP-Rab5 and GFP-Rab7. At 24h of tBid-RFP or mito-RFP coexpression with GFP-Rab5 or GFP-Rab7, fluorescence within ROI was bleached and fluorescence recovery analysed for $190 \mathrm{~s}$. (a) Image sequence shows a representative FRAP experiment of a cell coexpressing tBid-RFP and GFP-Rab7 before bleaching within ROI (white box) and during recovery. (b) Plot of fluorescence recovery of GFP-Rab5 at tBid-RFP-targeted mitochondria (tBid/Rab5), compared with recovery of cytoplasmic endosomal GFP-Rab5 in cells coexpressing mito-RFP (Con/Rab5). Standard deviations are shown as shaded regions. (c) Plot of GFP-Rab7 (tBid/Rab7) fluorescence recovery. Standard deviations are shown as shaded regions. At $24 \mathrm{~h}$ of tBid-RFP coexpression with GFP-Rab7, fluorescence within ROI was bleached for $190 \mathrm{~s}$ and fluorescence recovery analyzed. ' $n$ ' signifies the number of cells analyzed. (d) Coexpression of GFP-Rab7 or dominant-negative mutant GFP-Rab7(T22N) with tBid-RFP (24 h). (e) IF detection of Tom20 and Rabex-5 in tBid-RFP-expressing versus non-transfected neighboring cells. (f) Coexpression of RFP-Rabex-5 and tBid-GFP or mito-GFP (24 h). 3D surface renderings of RFP-Rabex-5 and tBid-GFP or mito-

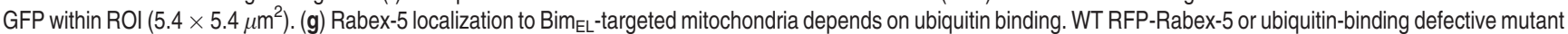

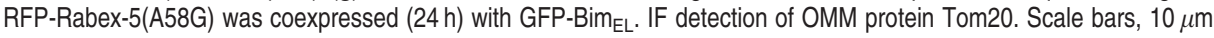



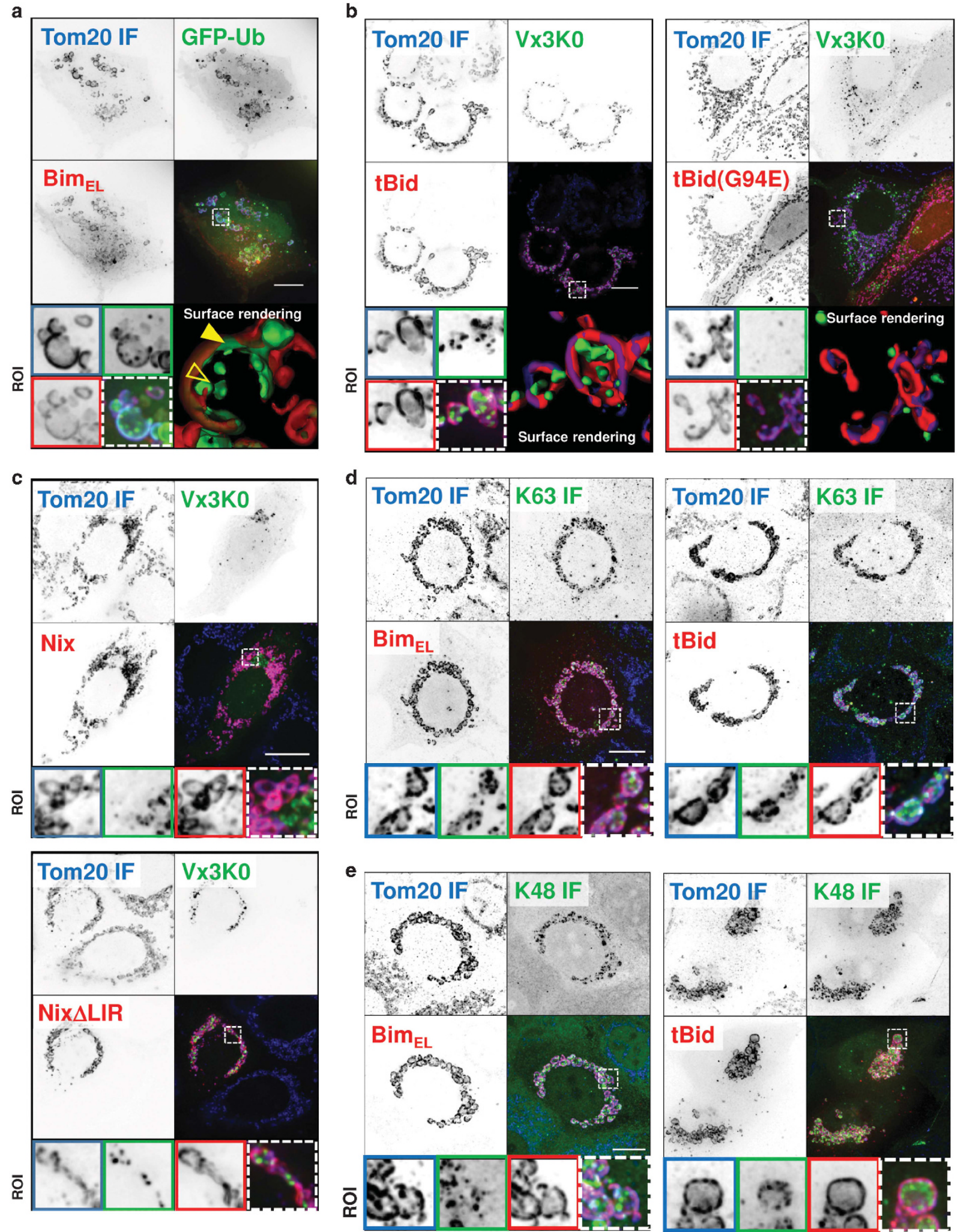

Figure 4 Canonical BH3-only protein-targeted mitochondria are ubiquitylated at the OMM and inside OMM-bound regions. (a) Coexpression (24 h) of GFP-Ub and RFPBim $_{\mathrm{EL}}$. IF detection of OMM marker Tom20. 3D surface rendering of GFP-Ub and RFP-Bim $\mathrm{EL}_{\mathrm{EL}}$-targeted mitochondria within ROI. (b) Coexpression (24 h) of K63 ubiquitin chain sensor Vx3K0-GFP with tBid-RFP or inactive-mutant tBid(G94E)-RFP, and Tom20 IF. 3D surface rendering of ROI. (c) Coexpression (24 h) of Vx3K0-GFP with either RFP-Nix

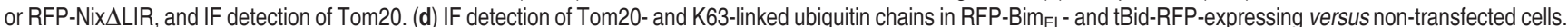
(e) IF detection of Tom20- and K48-linked ubiquitin chains in RFP-Bim $\mathrm{EL}^{-}$and tBid-RFP-expressing versus non-transfected cells. ROls, $5.4 \times 5.4 \mu \mathrm{m}^{2}$. Scale bars, $10 \mu \mathrm{m}$ 
a

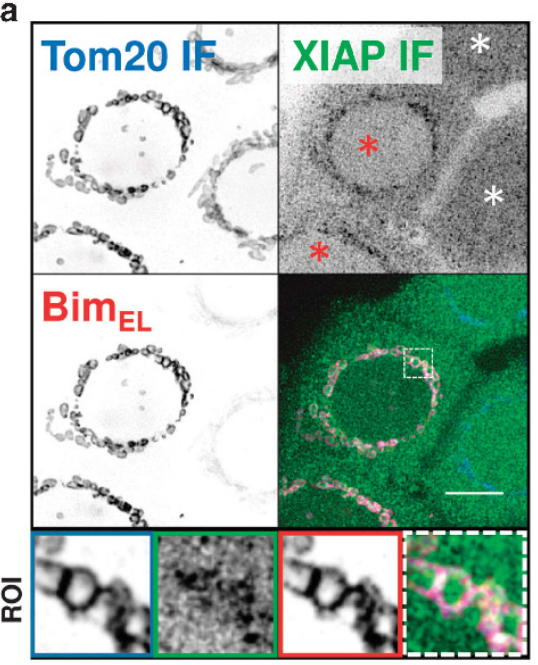

b

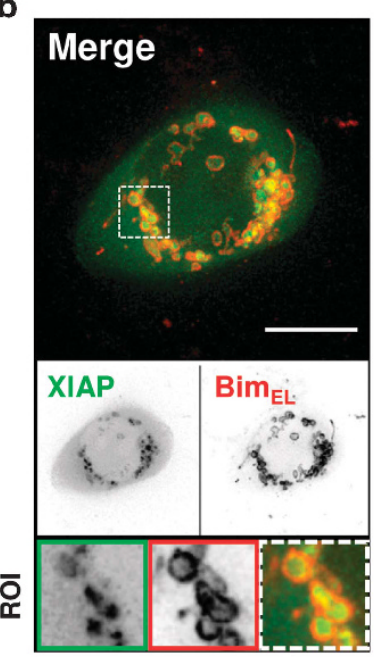

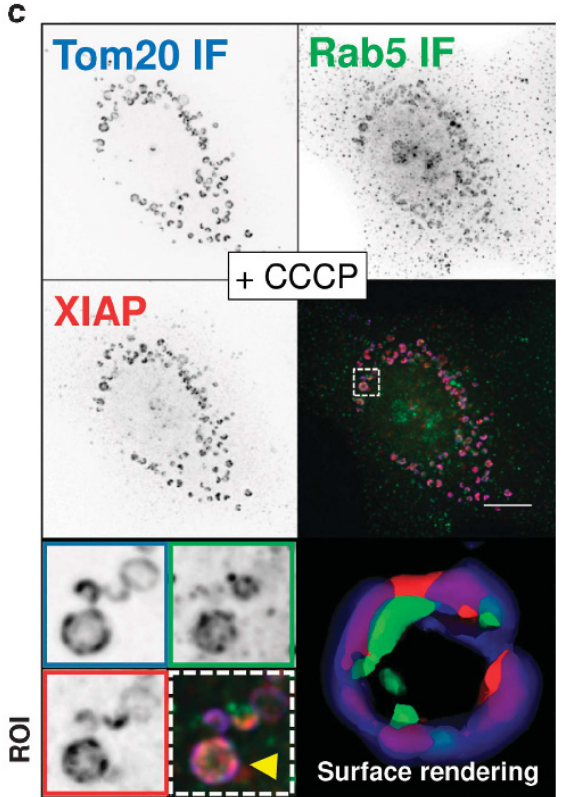

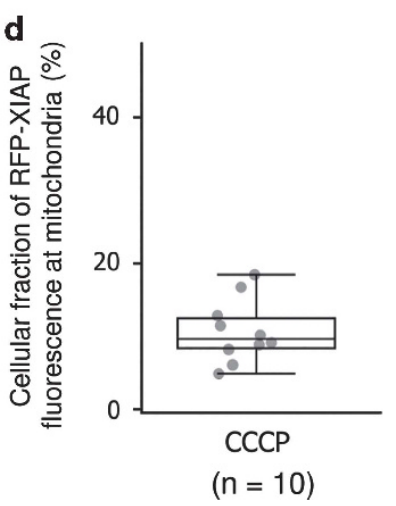

f

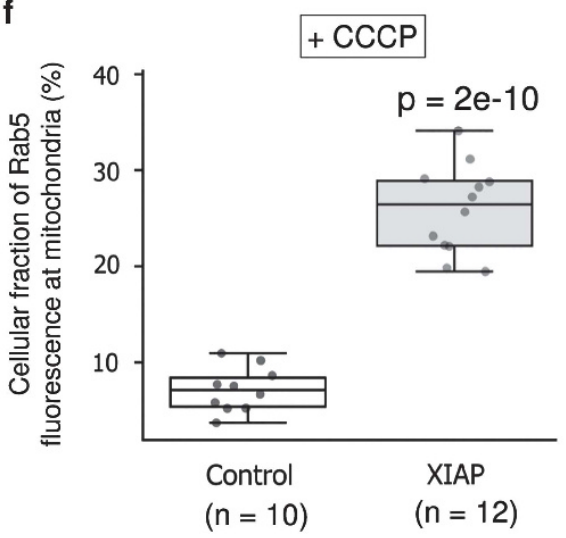

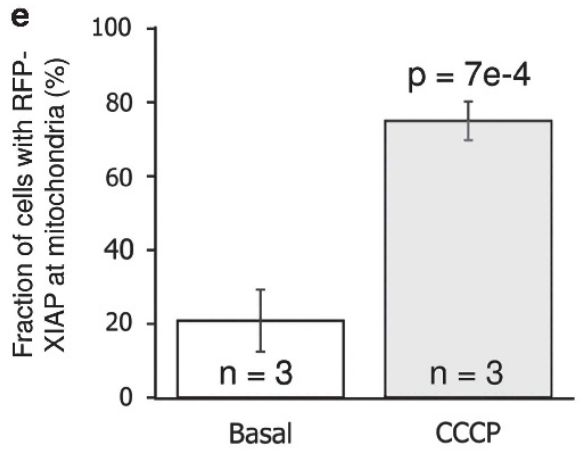

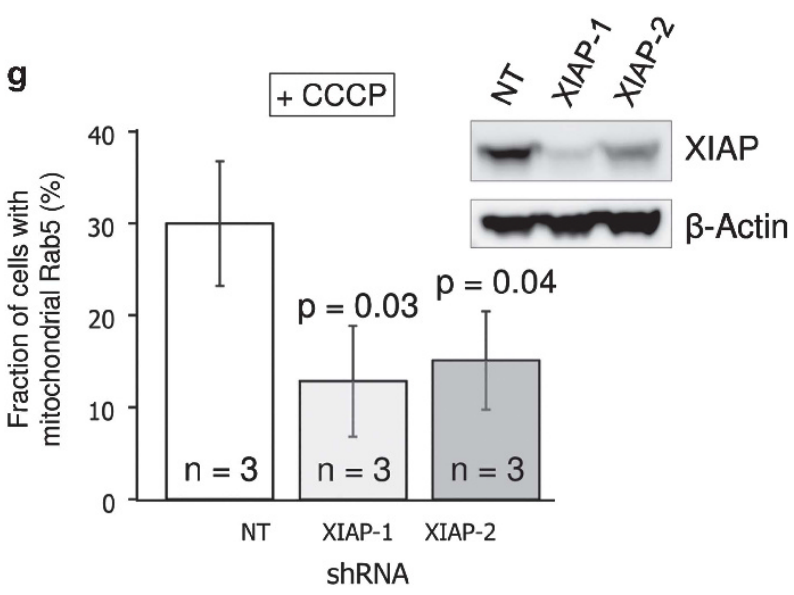

Figure 5 Mitochondrial depolarization induces mitochondrial translocation of XIAP and XIAP-dependent recruitment of endolysosomal trafficking machinery. (a) IF

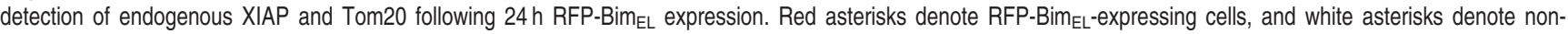
transfected cells. (b) Coexpression (24 h) of RFP-Bim $\mathrm{EL}$ and GFP-XIAP. (c) IF of Rab5 and Tom20 in RFP-XIAP-expressing cell following $3 \mathrm{~h}$ treatment with CCCP. 3D surface rendering of mitochondrion within ROI (yellow arrow). (a-c) Scale bars, $10 \mu \mathrm{m}$; ROls, $5.4 \times 5.4 \mu \mathrm{m}^{2}$. (d) Box plots represent cellular fraction of RFP-XIAP fluorescence colocalizing with Tom20-immunolabeled mitochondria following $3 \mathrm{~h}$ CCCP. (e) Bar graph represents fraction of cell population with mitochondrial RFP-XIAP following \pm CCCP. (f) Box plots show cellular fraction of endogenous Rab5 IF colocalizing with Tom20-labeled mitochondria following $3 \mathrm{~h}$ CCCP \pm XIAP overexpression. (g) Western blot demonstrates XIAP protein levels in MCF7 cells under stable expression of XIAP-targeting shRNAs (XIAP-1 and XIAP-2) and non-targeting shRNA (NT). Bar graph represents fraction of cell population with mitochondria-localized RFP-Rab5 fluorescence following \pm CCCP $(3 \mathrm{~h})$. ' $\mathrm{n}$ ' signifies the number of representative cells analyzed (c and $\mathbf{e}$ ) or experiments ( $\mathbf{d}$ and $\mathbf{f}$ ). P-values describe statistical significance versus control (Student's $t$-test) 

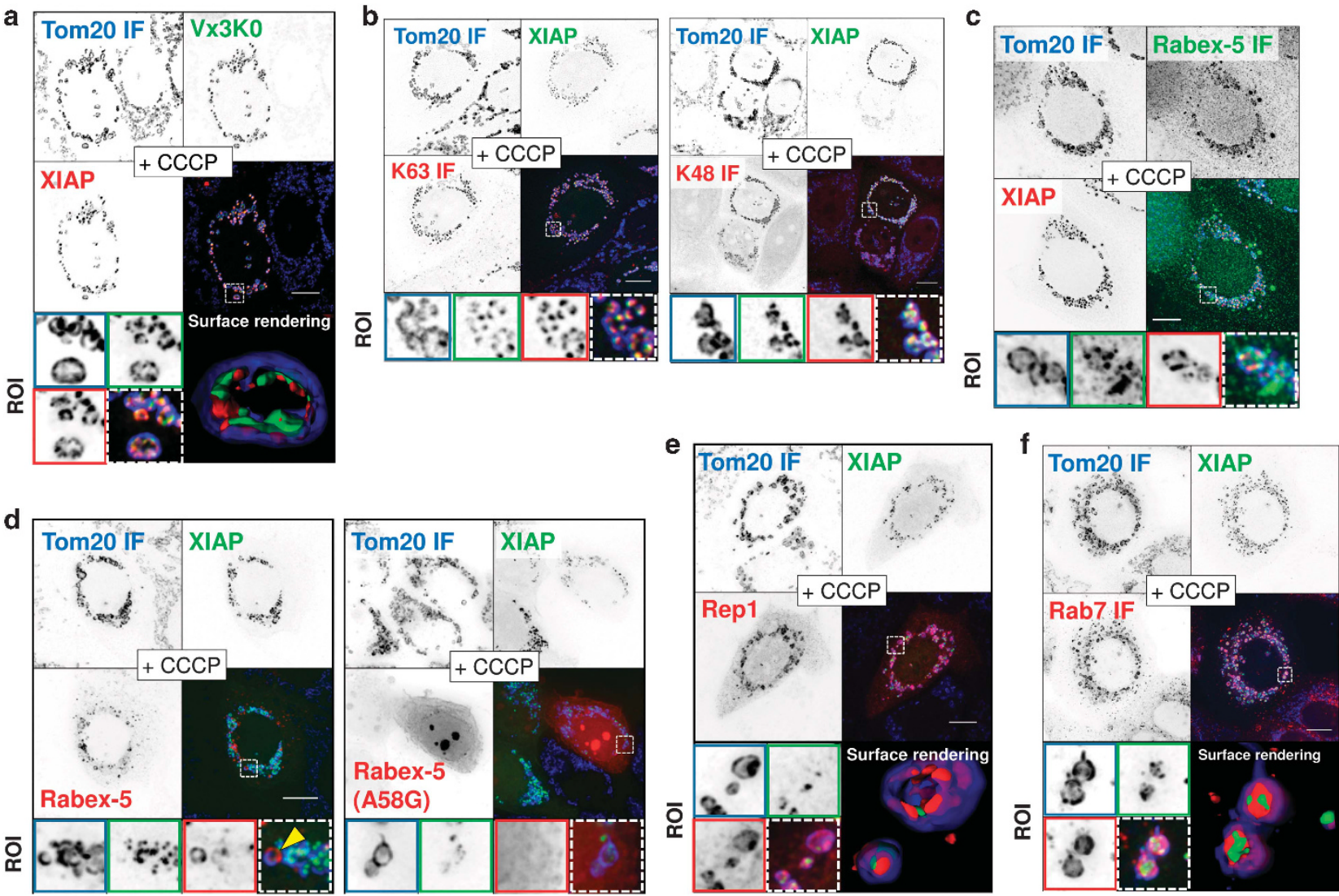

Figure 6 XIAP E3 ligase activity mediates ubiquitylation of depolarized mitochondria and endolysosomal targeting. (a) Coexpression of RFP-XIAP and K63 ubiquitin chain reporter Vx3K0-GFP, and treatment with $3 \mathrm{~h}$ CCCP. IF detection of Tom20. 3D surface rendering of ROI. (b) IF detection of Tom20-and K63-linked (left panel) or K48-linked (right panel) ubiquitin chains in RFP-XIAP- (green) versus non-transfected neighboring cells, following $3 \mathrm{~h}$ CCCP. (c) IF detection of endogenous Rabex-5 and Tom20 in RFP-XIAP-expressing cells treated with CCCP for $3 \mathrm{~h}$. (d) GFP-XIAP coexpression with either RFP-Rabex-5- or ubiquitin-binding mutant RFP-Rabex-5(A58G) and IF of Tom20. (e) RFP-Rep-1 coexpression with GFP-XIAP and Tom20 IF following 3 h CCCP. 3D surface rendering of ROI. (f) IF detection of endogenous Rab7 and Tom20 in RFP-XIAP- versus non-transfected neighboring cells, treated $3 \mathrm{~h}$ with CCCP. Overexpression experiments, $24 \mathrm{~h}$; ROls, $5.4 \times 5.4 \mu \mathrm{m}^{2}$; scale bars, $10 \mu \mathrm{m}$

(Figure 6d), whereas few RFP-Parkin-targeted mitochondria were sequestered within GFP-Rab7-labeled autophagolysosomes (Supplementary Figure S1d). Further, we excluded the possibility of Parkin participation in the XIAP-mediated pathway. Consistent with previous reports, ${ }^{48}$ we found that MCF7 cells are deficient in Parkin expression (Supplementary Figures S4e and f) and pathway induction does not lead to detectable Parkin levels at the mitochondria. Collectively, our data show that XIAP-mediated mitochondrial processing is distinguished from Parkin action by several parameters. XIAP action is all-or-nothing within the entire population of mitochondria, versus the slower action of sequestration-dependent mitophagy. ${ }^{13}$ Moreover, Parkin action was observed as spatially distinct from XIAP, acting solely at the OMM versus additional intramitochondrial targeting, respectively.

XIAP actuates Bax-mediated MOMP, independent of its E3 ligase activity. We next investigated the functional impact of XIAP-mediated mitochondrial processing on the apoptotic potential of mitochondria. CCCP alone or in combination with Parkin expression does not induce Bax activation. ${ }^{49}$ In contrast and unique to RFP-XIAP-expressing cells, CCCP treatment induced mitochondrial coating with endogenous Bax in OMM regions lacking Tom20, and Bax clustering at Tom20-labeled-OMM discontinuities (Figure 7a). These Tom20-void regions also localized with Rabex-5 (Figures $3 e$ and $6 \mathrm{~d}$ ), suggesting that endolysosomal entry into mitochondria integrates with the MOMP process. Interestingly, in cells expressing RFP-XIAP(F495L), deficient in ubiquitin transfer activity, ${ }^{50}$ CCCP similarly induced mitochondrial Bax clustering (Figure $7 \mathrm{~b}$ ), yet translocation of RFP-XIAP(F495L) to mitochondria was not observed. While CCCP-induced Bax activation was a hallmark feature of both WT and E3 ligase-mutated XIAP-expressing cells, important differences were observed between the release of cytochrome $c$ and Smac from WT and mutant XIAP-targeted mitochondria. Remarkably, following CCCP treatment, endogenous cytochrome $c$ was either fully retained in mitochondria containing RFP-XIAP and Bax clusters (Figure 7c) or released and/or degraded (Figure 7d). Similar heterogeneous results were obtained in response to RFPXIAP(F495L) (Figure 7d). However, quantification of total cellular cytochrome $c$ revealed a range of cytochrome $c$ levels following CCCP treatment, but no significant difference between WT and F495L XIAP (Figure 7e). 
a

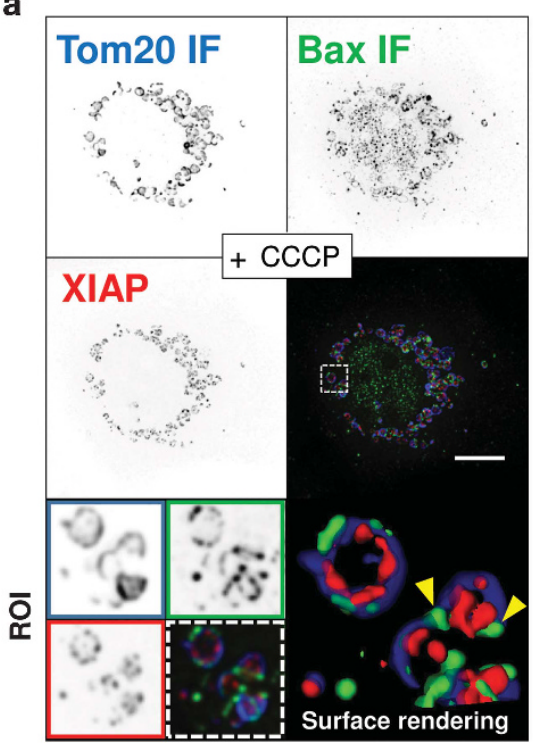

b

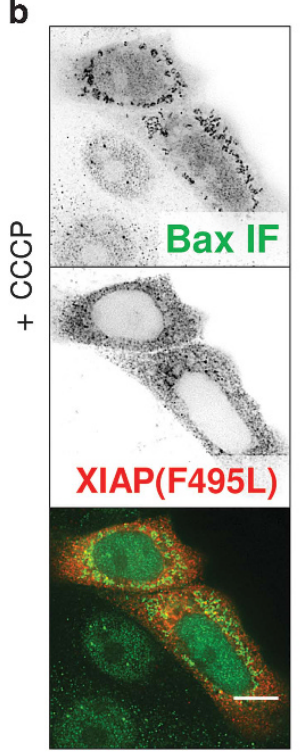

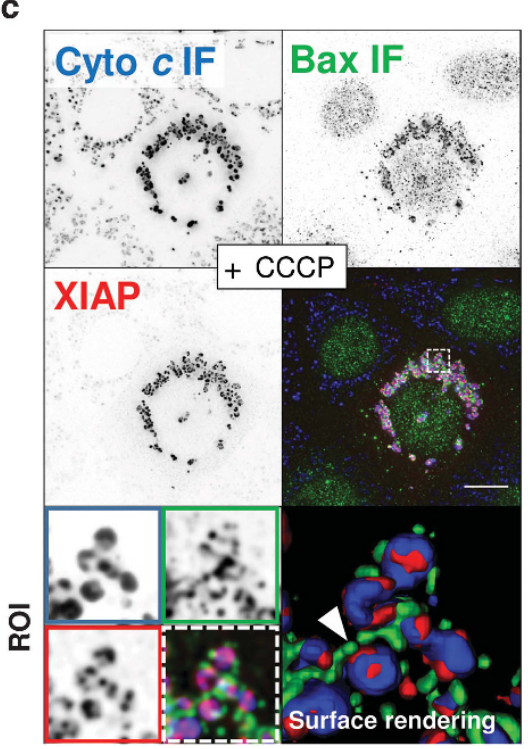
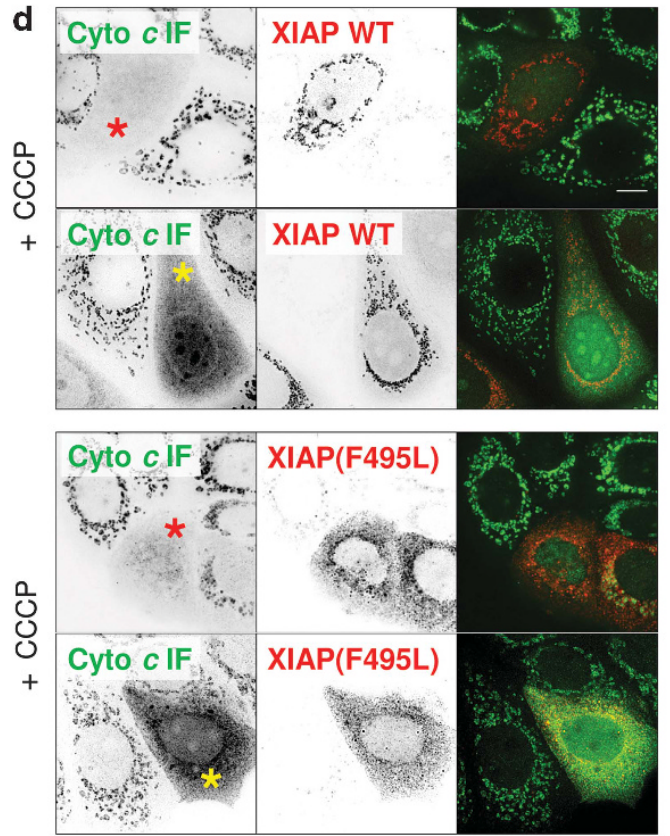

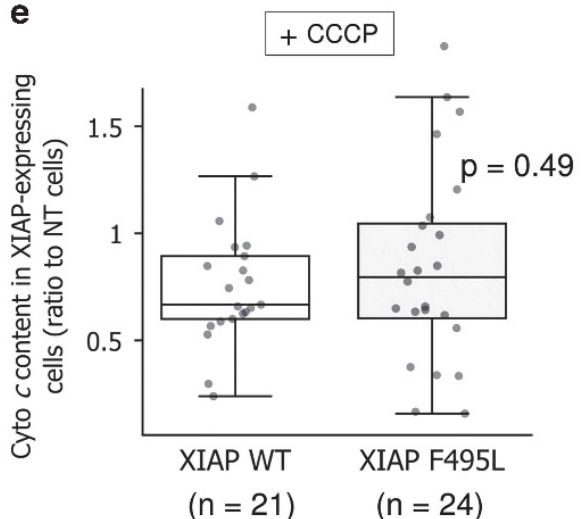

Figure 7 XIAP actuates MOMP of depolarized mitochondria. (a) Tom20 and Bax IF in RFP-XIAP-expressing cell treated for $3 \mathrm{~h}$ with CCCP. 3D rendering of ROI. Yellow arrows denote Bax clusters at Tom20-labeled OMM discontinuities. (b) Bax IF in E3 ligase-mutated RFP-XIAP(F495L)-expressing cells versus non-transfected cells, treated with CCCP for $3 \mathrm{~h}$. (c) Cytochrome $c$ (Cyto $c$ ) and Bax IF in RFP-XIAP- versus non-transfected cells, following $3 \mathrm{~h}$ treatment with CCCP. 3D rendering of ROI. White arrow points at exemplary Bax-targeted, cytochrome $c$-containing mitochondrion. (d) Cytochrome $c$ IF in cells expressing WT RFP-Xiap or E3 ligase mutant RFP-XIAP(495L), following $3 \mathrm{~h}$ CCCP. Red asterisk denotes degraded cytochrome $c$ phenotype; yellow asterisk denotes non-degraded, released cytochrome $c$ phenotype. (e) Quantification of cellular cytochrome $c$ content following $3 \mathrm{~h}$ CCCP. Box plots represent cellular cytochrome $c$ content in RFP-XIAP- versus RFP-XIAP(F495L)-expressing cells, relative to nontransfected (NT) cells. ' $n$ ' signifies the number of cells analyzed. P-value describes statistical significance between XIAP WT and F495L mutant (Student's $t$-test). Overexpression experiments, $24 \mathrm{~h}$; ROls, $5.4 \times 5.4 \mu \mathrm{m}^{2}$; scale bars, $10 \mu \mathrm{m}$

XIAP E3 ligase activity-mediated endolysosomal entry into mitochondria causes Smac degradation. In contrast to the above-described heterogeneous cellular cytochrome $c$ phenotypes, CCCP treatment induced a significant decrease in cellular Smac in RFP-XIAP WT cells (Figures $8 \mathrm{a}$ and $\mathrm{c}$ ). This effect was absent in CCCP-treated cells expressing RFP-XIAP(F495L) (Figure 8a). Instead, in RFP-XIAP(F495L) cells, released Smac was detected in the cytosol (Figure 8b). RFP-XIAP(F495L) induction of Smac release occurred in the absence of K63-chaining and Rab7 entry (Figure 8b), further connecting XIAP E3 ligasemediated mitochondrial ubiquitylation and endolysosomal entry with Smac degradation.

To address localization of Smac degradation by XIAP, we measured the response of mutants XIAP(W310A), which results in the loss of Smac binding to the XIAP BIR3 domain, ${ }^{51}$ 
and $\mathrm{XIAP}(\mathrm{D} 148 \mathrm{~A})$, which is unable to bind and inhibit caspase-3. ${ }^{52}$ Both XIAP(W310A) and XIAP(D148A) colocalized with mitochondrial compartments upon CCCP treatment (Figure 8c). Resulting Smac degradation was equivalent between XIAP(D148A) and WT XIAP, whereas XIAP(W310A) exhibited significantly reduced Smac degradation (Figure $8 \mathrm{C}$ ). Note, in cells in which XIAP(W310A) permeabilized mitochondria, Smac was observed either as colocalized in the mitochondria with $\mathrm{XIAP}(\mathrm{W} 310 \mathrm{~A})$ or as released and not degraded (data not shown). Taken together, these data suggest that Smac degradation takes place within the mitochondria and is dependent on a direct interaction between XIAP and Smac.

Finally, we investigated the pathway mediating Smac degradation. One hour pretreatment with either the proteasomal inhibitor epoxomicin (Epox) or lysosomal inhibitor bafilomycin A1 (BafA1) both resulted in significantly increased levels of cytosolic Smac following CCCP treatment (Figure 8d). Expression of the dominant-negative mutant Rab7(T22N) significantly increased Smac levels in cells with CCCP-induced mitochondrial RFP-XIAP (Figure 8e), confirming the role for endolysosomal control of Smac degradation.

\section{Discussion}

Maintaining physiologic levels of apoptosis requires safeguards, namely prosurvival $\mathrm{Bcl}-2$ member inhibition of MOMP, ${ }^{2}$ and IAP suppression of caspase activities. ${ }^{3,5}$ Among IAP members, XIAP is the most potent at limiting caspase activation, through inhibitory binding at BIR domains ${ }^{4,42}$ and E3 ligase-mediated degradation. ${ }^{43,53}$ As such, mitochondrial activation and/or amplification of apoptosis necessitates Smac release and subsequent binding to BIR2 and BIR3 domains of XIAP to inhibit caspase activities. ${ }^{6,54}$

Here, we report a novel function for XIAP that directly targets the apoptotic capacity of mitochondria. Our findings demonstrate that a fraction of XIAP localizes at dysfunctional, preapoptotic mitochondria, actuating MOMP and endolysosomal entry into inner mitochondrial compartments, and reducing Smac levels through lysosomal and proteasomal activities. Our data indicate that XIAP-mediated processing of mitochondria is a multistep process. The activation of MOMP was independent of its E3 ligase activity, whereas E3 ligasedependent signaling was required for XIAP translocation to and entry into the mitochondria, endolysosomal entry and Smac degradation (Figure 8f). These results integrate with past reports; during apoptosis XIAP binding of Smac can impede its release from the mitochondria ${ }^{44}$ and XIAP can ubiquitylate mature Smac in vitro. ${ }^{53,55}$ As mitochondrial factors, including Smac, are required in vivo for Bid-mediated apoptosis, ${ }^{6}$ we suggest that XIAP-mediated intramitochondrial degradation of Smac represents a novel apoptosis checkpoint, reducing the mitochondrial capacity for apoptosis activation and amplification.

The here-described mitochondrial action of XIAP is apparently independent of its direct caspase inhibitory function, as the caspase-binding mutant XIAP(D148A) targeted Smac to the same extent as WT XIAP. Furthermore, RING-bearing clAP1, which does not directly inhibit caspases, ${ }^{42}$ induced intramitochondrial K63 chains (Supplementary Figure S5a). As the RING-less Survivin ${ }^{42}$ did not translocate or affect mitochondrial K63 chaining (Supplementary Figure S5b), mitochondrial targeting of depolarized mitochondria may be a general mechanism for RING-bearing IAP members. Further, as XIAP knockdown suppressed, but did not eliminate, the basal activation of endosomal entry into the mitochondria in response to CCCP, it remains to be determined whether this is due to the action of other IAP family members or whether the mitochondriatargeting capacity of XIAP is concentration-dependent. ${ }^{44}$

Remarkably, canonical BH3-only proteins and LIR-mutated atypical $\mathrm{BH} 3-$ only proteins, Bnip3 and Nix, induced this pathway, independent of mitophagy induction. Moreover, XIAP targeting of mitochondria was mechanistically distinct and operating independently from both Parkin-mediated mitophagy and mitophagy-independent delivery of intramitochondrial components via vesicular transport to lysosomes. ${ }^{11}$ While damaged mitochondria can be selectively scavenged by mitophagy, ${ }^{8-10}$ the capacity of mitophagy to influence apoptosis is limited. Mitochondrial apoptosis transmits within a cell over minutes, ${ }^{20,21}$ yet mitophagy can require transcriptional upregulation, ${ }^{14,16,17,19}$ with mitochondrial elimination occurring progressively over days. ${ }^{15-17}$ In contrast, here we demonstrate that, concurrent with reduced Smac levels, cytochrome $c$ release was delayed in XIAP-and Bax-targeted mitochondria, indicating that XIAP can uncouple MOMP from apoptosis signaling, at least initially. Furthermore, the rapid nature of XIAP targeting of endolysosomes to the full

Figure 8 Intramitochondrial XIAP E3 ligase activity causes degradation of Smac. (a) RFP-XIAP WT- or RFP-XIAP(F495L)-expressing cells were treated with CCCP for $3 \mathrm{~h}$. Box plots represent cellular Smac content, normalized to non-transfected (NT) control cells. $P$-value describes statistical significance compared with XIAP WT (Student's t-test). (b) Coexpression of XIAP E3 ligase-inactive mutant RFP-XIAP(F495L) with K63-chain marker Vx3K0-GFP or GFP-Rab7. IF detection of Smac following 3h CCCP. Yellow asterisks denote non-degraded, released Smac phenotype. Scale bar, $10 \mu \mathrm{m}$. (c) GFP-XIAP WT, caspase-3 binding-deficient GFP-XIAP(D148A) or Smac bindingdeficient GFP-XIAP(W310A) were treated with CCCP for $3 \mathrm{~h}$ and immunostained for Smac. Box plots represent cellular Smac levels, normalized to non-transfected (NT) control cells. P-value describes statistical significance compared with GFP-XIAP WT (Student's $t$-test). (d) GFP-XIAP-expressing cells were pretreated $1 \mathrm{~h}$ with DMSO, BafA1 $(100 \mathrm{nM})$ or Epox $(1 \mu \mathrm{M})$. Following, cells were treated for $3 \mathrm{~h}$ with CCCP, CCCP/Epox or CCCP/Baf. Box plots represent cellular Smac levels, normalized to NT control cells. $P$-values describe statistical significance compared with CCCP alone (Student's $t$-test). (e) Coexpression of RFP-XIAP and GFP-Rab7 WT or dominant-negative mutant GFPRab7(T22N). Treatment with CCCP for $3 \mathrm{~h}$ followed by IF detection of Smac. Box plots represent cellular Smac levels, normalized to NT control cells. P-values describe statistical significance compared with GFP-Rab7 WT (Student's $t$-test). For (a and $\mathbf{c}-\mathbf{e}$ ), ' $n$ ' signifies the number of cells analyzed. (f) Schematic describing proposed interactions between XIAP E3 ligase activity and endosomal trafficking. In response to CCCP-induced loss of mitochondrial membrane potential, (1) XIAP activates Bax at the OMM in an E3 ligase-independent manner. (2) Dependent on E3 ligase activity, XIAP targets the GEF Rabex-5 to the OMM. XIAP (3) translocates to inner mitochondrial compartments, where it (4) induces prominent K63 and K48 polyubiquitylation. (5) Concurrently, XIAP E3 ligase activity induces Rab5 and Rab7 entry into mitochondria. (6) Intramitochondrial XIAP induces Smac degradation in a manner dependent on XIAP binding to Smac, and proteasomal and lysosomal activities. The green region of the OMM denotes permeabilization events. EE, early endosome; IMS, mitochondrial intermembrane space; LE, late endosome. Overexpression experiments, $24 \mathrm{~h}$ 
mitochondrial population contrasts fractional targeting of mitochondria by mitophagy, revealing a novel, potent function for endolysosomes in programmed cell death.
As an upstream regulator during apoptosis and mitochondrial depolarization, our findings implicate the guanine nucleotide exchange factor Rabex-5. It was recently shown a

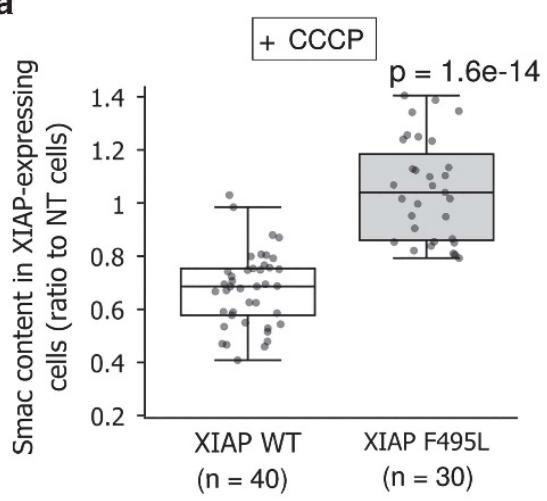

C
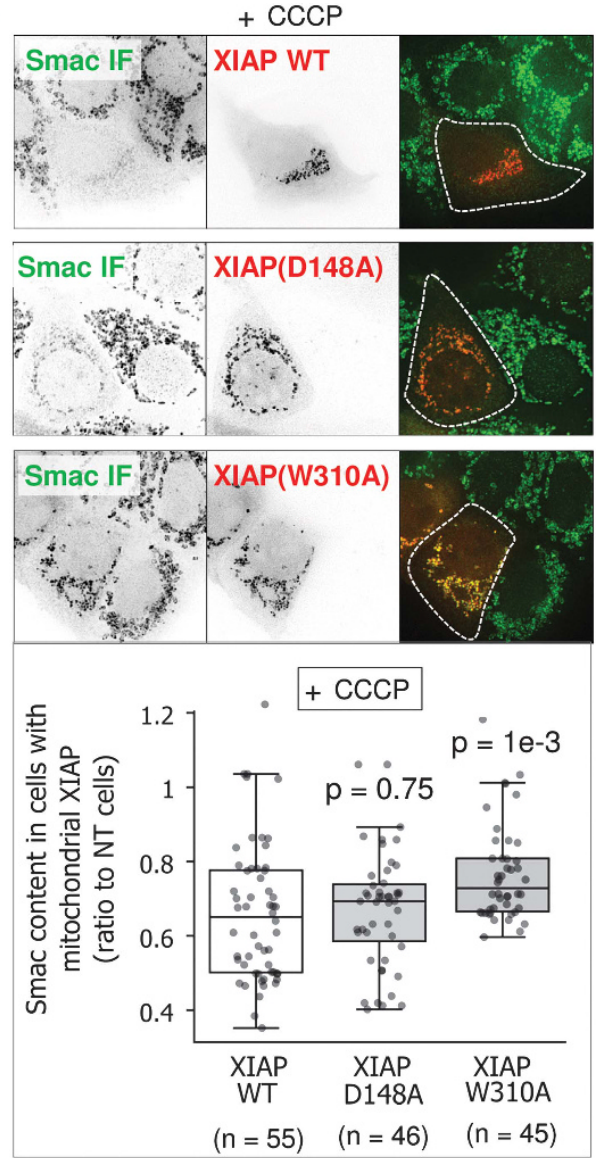

Mitochondrion

f

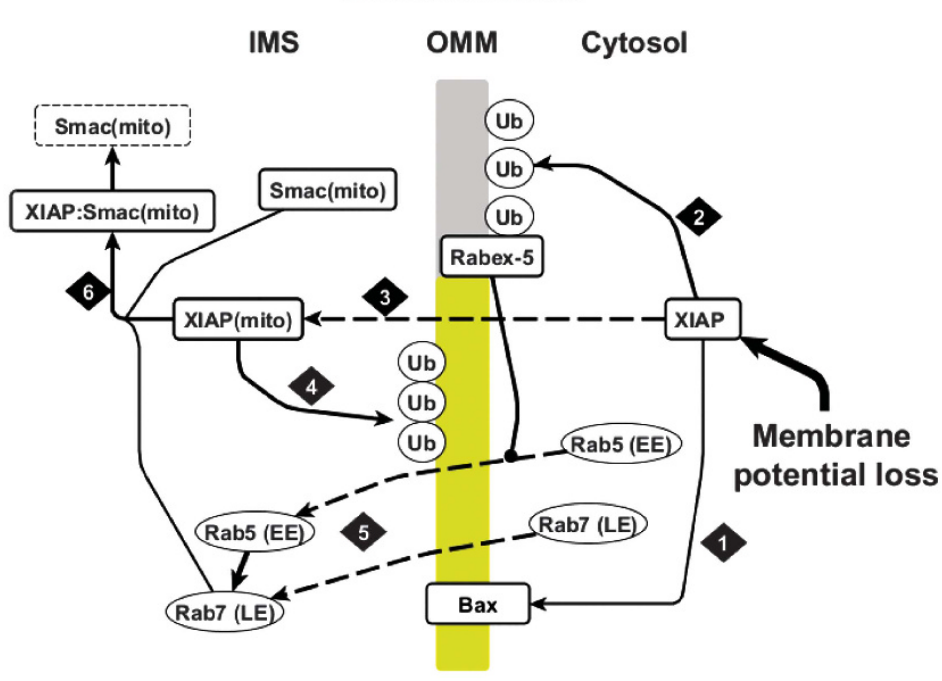

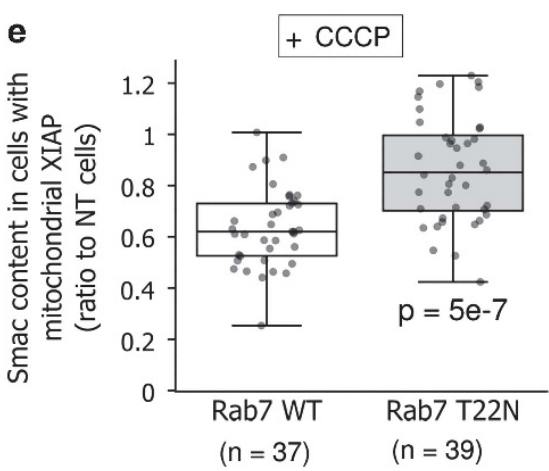


that acute targeting of Rabex-5 to mitochondria efficiently corecruits Rab5 in a rapid ( $\sim 10 \mathrm{~min})$ manner. ${ }^{35}$ Notably, using this described ${ }^{35}$ forced localization of Rabex-5 to mitochondria was not sufficient to activate MOMP (data not shown). Of note, mitochondria targeted by canonical $\mathrm{BH} 3-$ only proteins, or XIAP under conditions of CCCP, showed increased diameters (Supplementary Figure S3c). We focused our analysis on larger individual mitochondria (as shown in ROIs), which enabled us to conclude localization confidently, at and within the mitochondria. Future in-depth studies using super-resolution and EM imaging will be required to delineate the specific interactions and kinetics of endolysosomal machineries and MOMP activities.

Overall, our data reveal an increased complexity to lysosomal crosstalk with the apoptosis regulatory machinery during bioenergetics stress. Of note, a recent study reported that XIAP rapidly activates MOMP, and consequently apoptosis, in response to loss of extracellular matrix signaling, ${ }^{56}$ suggesting that this pathway might be regulated in both a prosurvival and prodeath manner. Moreover, as bioenergetic stresses can occur during ischemic injury ${ }^{57}$ and are rapidly propagated within mitochondrial populations through calcium and oxidative signaling, ${ }^{58,59}$ future work will address the functionality of mitochondriatargeted XIAP before and during apoptosis, as well as the capacity of XIAP to precondition cells before apoptosis induction.

\section{Materials and Methods \\ Materials. Cell culture reagents were obtained from Invitrogen (Darmstadt, Germany), Sigma (Steinheim, Germany), Lonza (Walkersville, MD, USA) and PAN Biotech (Aidenbach, Germany). Electron microscopy-grade paraformaldehyde was obtained from EMS (Hatfield, PA, USA). BafA1 was purchased from Enzo Lifesciences (Lörrach, Germany). DMSO was from Genaxxon Biosciences (Ulm, Germany). Epox and CCCP were obtained from Merck Millipore (Darmstadt, Germany).}

Plasmids. Human cIAP1, Rabex-5, Rep-1, Survivin and XIAP were cloned as $\mathrm{N}$-terminal tagRFP fusion proteins. Human Bad, Bim $\mathrm{EL}_{\mathrm{L}}, \mathrm{Rab5}$ and XIAP were cloned as N-terminal GFP fusion proteins. Canine Rab7 was cloned as an $\mathrm{N}$-terminal mCherry fusion protein. Human tBid, cleavage site Asp-60, was cloned as C-terminal mCherry and GFP fusion proteins and tBid(G94E), cleavage site Asp-75, was cloned as a C-terminal mCherry fusion protein. Parkin from the mCherry-Parkin (Addgene, Cambridge, MA, USA; plasmid 23956) was subcloned as tagRFP-Parkin. Additional protein expression vectors were used as described previously: GFP-Bik (Addgene; plasmid 10952), GFP-Ub (Addgene; plasmid 11928), Vx3K0-GFP (Addgene; plasmid 35527), WT and T22N GFP-Rab7 ${ }^{29}$ MitoGFP/RFP, GFP-LC3B, GFP-GATE-16, WT and $\Delta$ LIR mCherry-Nix and mCherryBnip3 were as described previously. ${ }^{16,17}$ In text and figure legends, tagRFP and mCherry are both referred to as RFP. Indicated mutants were generated by sitedirected mutagenesis.

Cell Culture and lentivirus-mediated gene knockdown. Human MCF7 breast (Cell Line Services, Eppelheim, Germany) and embryonic kidney $293 \mathrm{~T}$ cell lines were maintained in DMEM supplemented with $10 \% \mathrm{FBS}$, L-glutamine, non-essential amino acids and penicillin/streptomycin/amphotericin B. For lentiviral gene knockdown, pLKO.1 shRNA was generated against human XIAP target sequences: $5^{\prime}$-AGCTGTAGATAGATGGCAATA-3' (XIAP-1) and $5^{\prime}$-GCACTCCAACTTCTAATCAAA-3' (XIAP-2). pLKO lentiviral vectors containing shRNA were transfected into 293T cells together with psPAX2 (packaging vector) and PMD2.G (VSV-G envelope protein expression vector) using calcium phosphate transfection (Sigma). MCF7 cells were infected with virus-containing supernatants at $50 \%$ confluence and cells selected and maintained with puromycin (Sigma; $1 \mu \mathrm{g} / \mathrm{ml}$ ). Once plated for experiments, cells were kept in puromycin-free medium.
Gene expression and drug treatments. Transient transfections were performed using jetPRIME transfection reagent (Peqlab, Erlangen, Germany). Experiments were performed at 24 or $48 \mathrm{~h}$ of expression, as indicated. All treatments with CCCP, Epox and/or BafA1 were performed in fully supplemented cell culture medium.

Immunostaining. MCF7 cells were plated in 8-well microscopy $\mu$-slides (ibidi, Munich, Germany), transfected and treated as indicated, and imaged live or following paraformaldehyde (PFA) fixation (4\% PFA in PBS, pH 7.4). For N-SIM experiments, cells were plated in 8-well, glass-bottom $\mu$-slides (170 $\mu \mathrm{m}$, ibidi). For visualization of endogenous proteins, fixed cells were permeabilized with $0.3 \%$ Triton X-100 in PBS and blocked with 3\% BSA. Cells were then incubated with primary antibodies against cytochrome $c$ (Santa Cruz Biotechnology, Santa Cruz, CA, USA; no. sc-13156), Parkin (Santa Cruz Biotechnology; no. sc-32282), Rabex-5 (Santa Cruz Biotechnology; no. sc-68344), Smac/DIABLO (Santa Cruz Biotechnology; no. sc-22766), Tom20 (Santa Cruz Biotechnology; no. sc-11415 or no. sc-17764), TRAP1 (Novus Biologicals, Littleton, CO, USA; no. NB300-555), ubiquitin, K48-linkage-specific (Merck Millipore; no. 05-1307), or ubiquitin, K63linkage-specific (Merck Millipore; no. 05-1308) at room temperature for $1 \mathrm{~h}$, or with Rab5 (Cell Signaling; no. 3547), Rab7 (Cell Signaling, Beverly, MA, USA; no. 9367) or XIAP (Santa Cruz Biotechnology; no. sc-55551) antibodies at $4{ }^{\circ} \mathrm{C}$ overnight. Fluorescent staining was performed for $30 \mathrm{~min}$ at room temperature using highly cross-absorbed Alexa Fluor 405, 488, 546 or 647 secondary antibodies (Invitrogen).

Widefield fluorescence imaging. Widefield fluorescence microscopy was performed with a DeltaVision RT microscope system (Applied Precision, Issaquah, WA, USA) using a $\times 60$ oil immersion objective. Z-stacks of representative cells were captured with $0.3 \mu \mathrm{m}$ increments and deconvolved (Softworx, Applied Precision). A subset of Z-stacks were captured at $0.15 \mu \mathrm{m}$ step increments, as indicated in text. Image analysis and preparation was performed using ImageJ (http://rsbweb.nih.gov/ij) and Fiji (http://fiji.sc/Fiji). Displayed images of representative cells are total intensity projections of Z-stacks, or single slices, as indicated in text. Single-color channels are shown in inverted gray scale for enhanced visibility of details. Pseudocolors in merged color images correspond to font colors of protein labels within single-color images. IF of endogenous proteins is indicated within single-color images by 'IF', to discriminate from detection of fluorescent protein-tagged proteins. 3D surface renderings of $\mathrm{ROI}$ were prepared using the Image J $3 \mathrm{D}$ Viewer plugin. In 3D surface renderings displaying three-color channels, blue Tom20-IF is set at $50 \%$ transparency.

Quantifications of fluorescence microscopy data. Quantification of population responses. At $24 \mathrm{~h}$ of expression, cells were treated for $3 \mathrm{~h}$ with CCCP $(20 \mu \mathrm{M})$, fixed and immunostained with $\alpha$-Tom20 to visualize OMM. Per condition, $\geq 100$ cells were scored for mitochondrial translocation of RFP-XIAP or RFPRab5, in three independent experiments.

Quantification of Rab5 and Rab7 distribution: From Z-stacks, single cells were cropped for analysis by ImageJ. Binary masks for each slice within $\geq 10$ representative Z-stacks per condition in three independent experiments of (i) Tom20-labeled mitochondria and (ii) fluorescent protein-tagged Rab5 or Rab7 were generated by image segmentation. Slice-by-slice colocalization of mitochondrial and endosome masks were calculated using the Boolean AND function. All slices for each binary stack were summed, and the ratio of area calculated from mitochondrially localized Rab over total endosomal Rab is reported as a cellular fraction.

Quantification of XIAP translocation: From Z-stacks, single cells were cropped for analysis by ImageJ. Binary masks for each slice within Z-stacks of (1) total cell area and (2) mitochondrial XIAP were generated by image segmentation. Masks were used to calculate slice-by-slice cellular and mitochondrial XIAP, respectively. All slices for each stack were summed, and the ratio of total mitochondrial XIAP to total cellular XIAP fluorescence is reported.

Quantification of cellular cytochrome $c$ and Smac levels: Images were acquired using the Z-sweep function to capture total cellular fluorescence within a single image, using identical exposure settings. Identical cytosolic areas within CCCPtreated cells, with and without WT or mutant XIAP expression, were analyzed. Per cell, cytosolic regions were measured for total cytochrome $c$ or Smac intensity. Per condition, the ratio of cytochrome $c / S m a c$ levels in cells with expressing XIAP cells versus cytochrome c/Smac levels in non-transfected cells was calculated. Per condition, $>30$ representative cells were analyzed, from at least three independent experiments. Data were plotted using http://plot.ly. 
Fluorescence recovery after photobleaching. FRAP experiments were performed with a Leica SP5 Laser Scanning Confocal Microscope (Leica Microsystems, Wetzlar, Germany) equipped with a $\times 60$ oil immersion objective. At $24 \mathrm{~h}$ after transfection, a region of tBid-RFP-targeted mitochondria was bleached of GFP and RFP fluorescence using maximal intensity $488 \mathrm{~nm}$ laser. Fluorescence recovery of bleached mitochondria was monitored every $10 \mathrm{~s}$ for $190 \mathrm{~s}$ and ROls were analyzed using Leica LAS AF Lite software (Leica Microsystems). Recovery values were normalized and plotted using http://plot.ly.

Super-resolution microscopy. Super-resolution microscopy was performed using a Nikon-structured illumination microscope (N-SIM; Nikon Imaging Center, Heidelberg, Germany). Images were acquired on a Nikon Ti inverted microscope (Nikon, Düsseldorf, Germany), using 488 and $561 \mathrm{~nm}$ laser excitation, and a Nikon Apo TIRF $\times 100$ NA 1.49 oil immersion objective (Nikon). Z-stacks were collected at $0.15 \mu \mathrm{m}$ steps, reconstructed using NIS-Elements (http:// www.nikoninstruments.com), and analyzed using Fiji.

Electron microscopy. MCF7 cells were transfected with the indicated plasmids and at $24 \mathrm{~h}$ (tBid, mito-RFP) or $48 \mathrm{~h}$ (Nix, Nix $\Delta \mathrm{LIR}$ ) after transfection, fixed and prepared for cryosectioning as detailed in Ghosh et al. ${ }^{60}$ Thawed cryosections were labeled with antibodies against mRFP ${ }^{61}$ (kind gift from Dr. Barbara Mueller, Institute of Infectious Diseases, University of Heidelberg, Heidelberg, Germany), GFP (Clontech, Mountain View, CA, USA), or Tom20 (Santa Cruz Biotechnology; no. sc-11415) as detailed in Slot et al. ${ }^{62}$ Protein A coupled to $10 \mathrm{~nm}$ gold particles was purchased from the University of Utrecht, Utrecht, The Netherlands. Images were acquired at 25000 to 40000 magnification with a Zeiss EM10 electron microscope (Zeiss, Oberkochen, Germany) equipped with a CCD digital camera, and images prepared with ImageJ.

Western blotting. MCF7 whole-cell lysates were prepared with RIPA lysis buffer (Millipore, Temecula, CA, USA) containing Complete protease inhibitor cocktail (Roche, Mannheim, Germany). Protein concentrations were determined using Coomassie reagent (Sigma). Samples were electrophoresed using Bis-Tris NuPAGE gels (Invitrogen) and proteins were transferred to nitrocellulose using the iBlot dry blotting system (Invitrogen). Immunodetection was performed using antibodies against $\beta$-actin (GeneTex, Irvine, CA, USA; no. GTX26276) and XIAP (Santa Cruz Biotechnology; no. sc-55551). Horseradish peroxidase-conjugated secondary antibodies (Cell Signaling) were used for digital chemiluminescence detection (Intas, Göttingen, Germany). Blot shown is representative of three independent experiments.

Statistical analyzes. The probability of statistically significant increases or decreases between conditions of at least three independent experiments was determined using the Student's $t$-test. Two-tailed, unpaired $t$-tests were performed. Values are expressed for bar graphs as mean \pm S.D. Box plots show individual data points, median values and whiskers indicate range of data points. Statistical significances and number of measurements are indicated in each figure.

Acknowledgements. For constructs, we would like to acknowledge Drs. B van Deurs (Copenhagen University), R Youle (Addgene; plasmid 23956), B Fang (Addgene; plasmid 10952), N Dantuma (Addgene; plasmid 11928) and R Cohen (Addgene; 35527). We thank Dr. C Ackermann of the Nikon Imaging Center Heidelberg for assistance with N-SIM. This work was supported through SBCancer within the Helmholtz Alliance on Systems Biology funded by the Initiative and Networking Fund of the Helmholtz Association (NRB); and the e:Bio Grant No 0316191 (LysoSys) of the Federal Ministry of Education and Research (BMBF), Germany $(\mathrm{AH}-\mathrm{B})$. The funders had no role in study design, data collection and analysis, decision to publish or preparation of the manuscript.

\section{Author Contributions}

$\mathrm{AH}-\mathrm{B}$ and NRB conceived and designed the study, performed experiments, analyzed the data and prepared figures. JKL performed electron microscopy experiments. SCC contributed intellectually and edited the manuscript. AH-B and NRB wrote the manuscript.

1. Ni Chonghaile T, Sarosiek KA, Vo TT, Ryan JA, Tammareddi A, Moore Vdel G et al. Pretreatment mitochondrial priming correlates with clinical response to cytotoxic chemotherapy. Science 2011; 334: 1129-1133.
2. Kroemer G, Galluzzi L, Brenner C. Mitochondrial membrane permeabilization in cell death. Physiol Rev 2007; 87: 99-163.

3. Deveraux QL, Reed JC. IAP family proteins - suppressors of apoptosis. Genes Dev 1999; 13: 239-252.

4. Galban S, Duckett CS. XIAP as a ubiquitin ligase in cellular signaling. Cell Death Differ 2010; 17: 54-60.

5. Vaux DL, J Silke. IAPs RINGs and ubiquitylation. Nat Rev Mol Cell Biol 2005; 6: 287-297.

6. Jost PJ, Grabow S, Gray D, McKenzie MD, Nachbur U, Huang DC et al. XIAP discriminates between type I and type II FAS-induced apoptosis. Nature 2009; 460: 1035-1039.

7. Marino G, Niso-Santano M, Baehrecke EH, Kroemer G. Self-consumption: the interplay of autophagy and apoptosis. Nat Rev Mol Cell Biol 2014; 15: 81-94.

8. Youle RJ, Narendra DP. Mechanisms of mitophagy. Nat Rev Mol Cell Biol 2011; 12: 9-14.

9. Lemasters JJ, Nieminen AL, Qian T, Trost LC, Elmore SP, Nishimura Y et al. The mitochondrial permeability transition in cell death: a common mechanism in necrosis, apoptosis and autophagy. Biochim Biophys Acta 1998; 1366: 177-196.

10. Soubannier V, McLelland GL, Zunino R, Braschi E, Rippstein P, Fon EA et al A vesicular transport pathway shuttles cargo from mitochondria to lysosomes. Curr Biol 2012; 22: 135-141.

11. McLelland GL, Soubannier V, Chen CX, McBride HM, Fon EA. Parkin and PINK1 function in a vesicular trafficking pathway regulating mitochondrial quality control. EMBO J 2014; 33: 282-295.

12. Narendra D, Tanaka A, Suen DF, Youle RJ. Parkin is recruited selectively to impaired mitochondria and promotes their autophagy. J Cell Biol 2008; 183: 795-803.

13. Geisler S, Holmstrom KM, Skujat D, Fiesel FC, Rothfuss OC, Kahle PJ et al. PINK1/Parkinmediated mitophagy is dependent on VDAC1 and p62/SQSTM1. Nat Cell Biol 2010; 12 : 119-131.

14. Liu L, Feng D, Chen G, Chen M, Zheng $Q$, Song $P$ et al. Mitochondrial outer-membrane protein FUNDC1 mediates hypoxia-induced mitophagy in mammalian cells. Nat Cell Biol 2012; 14: 177-185.

15. Hamacher-Brady A, Brady NR, Logue SE, Sayen MR, Jinno M, Kirshenbaum LA et al. Response to myocardial ischemia/reperfusion injury involves Bnip3 and autophagy. Cell Death Differ 2007; 14: 146-157.

16. Novak I, Kirkin V, McEwan DG, Zhang J, Wild P, Rozenknop A et al. Nix is a selective autophagy receptor for mitochondrial clearance. EMBO Rep 2010; 11: 45-51.

17. Zhu Y, Massen S, Terenzio M, Lang V, Chen-Lindner S, Eils R et al. Modulation of serines 17 and 24 in the LC3-interacting region of Bnip3 determines pro-survival mitophagy versus apoptosis. J Biol Chem 2013; 288: 1099-1113.

18. Hanna RA, Quinsay MN, Orogo AM, Giang K, Rikka S, Gustafsson AB. Microtubule-associated protein 1 light chain 3 (LC3) interacts with Bnip3 protein to selectively remove endoplasmic reticulum and mitochondria via autophagy. J Biol Chem 2012; 287: 19094-19104.

19. Sandoval H, Thiagarajan P, Dasgupta SK, Schumacher A, Prchal JT, Chen M et al. Essential role for Nix in autophagic maturation of erythroid cells. Nature 2008; 454: 232-235.

20. Garcia-Perez C, Roy SS, Naghdi S, Lin X, Davies E, Hajnoczky G. Bid-induced mitochondrial membrane permeabilization waves propagated by local reactive oxygen species (ROS) signaling. Proc Natl Acad Sci USA 2012; 109: 4497-4502.

21. Rehm M, Huber HJ, Hellwig CT, Anguissola S, Dussmann H, Prehn JH. Dynamics of outer mitochondrial membrane permeabilization during apoptosis. Cell Death Differ 2009; 16: 613-623.

22. Kuwana T, Bouchier-Hayes L, Chipuk JE, Bonzon C, Sullivan BA, Green DR et al. $\mathrm{BH} 3$ domains of $\mathrm{BH} 3$-only proteins differentially regulate Bax-mediated mitochondrial membrane permeabilization both directly and indirectly. Mol Cell 2005; 17: 525-535.

23. Kim H, Rafiuddin-Shah M, Tu HC, Jeffers JR, Zambetti GP, Hsieh JJ et al. Hierarchical regulation of mitochondrion-dependent apoptosis by BCL-2 subfamilies. Nat Cell Biol 2006; 8: 1348-1358.

24. Zhang J, Ney PA. Role of BNIP3 and NIX in cell death, autophagy, and mitophagy. Cell Death Differ 2009; 16: 939-946.

25. Janicke RU, Sprengart ML, Wati MR, Porter AG. Caspase-3 is required for DNA fragmentation and morphological changes associated with apoptosis. J Biol Chem 1998; 273: 9357-9360.

26. Yang S, Thor AD, Edgerton S, Yang X. Caspase-3 mediated feedback activation of apical caspases in doxorubicin and TNF-alpha induced apoptosis. Apoptosis 2006; 11: 1987-1997.

27. Luo S, Rubinsztein DC. Apoptosis blocks Beclin 1-dependent autophagosome synthesis: an effect rescued by Bcl-xL. Cell Death Differ 2010; 17: 268-277.

28. Klionsky DJ, Abdalla FC, Abeliovich $\mathrm{H}$, Abraham RT, Acevedo-Arozena A, Adeli $\mathrm{K}$ et al. Guidelines for the use and interpretation of assays for monitoring autophagy. Autophagy 2012; 8: 445-544.

29. Bucci C, Thomsen P, Nicoziani P, McCarthy J, van Deurs B. Rab7: a key to lysosome biogenesis. Mol Biol Cell 2000; 11: 467-480.

30. Rink J, Ghigo E, Kalaidzidis Y, Zerial M. Rab conversion as a mechanism of progression from early to late endosomes. Cell 2005; 122: 735-749.

31. Wang K, Yin XM, Chao DT, Milliman CL, Korsmeyer SJ. BID: a novel BH3 domain-only death agonist. Genes Dev 1996; 10: 2859-2869.

32. Jordens I, Fernandez-Borja M, Marsman M, Dusseljee S, Janssen L, Calafat J et al. The Rab7 effector protein RILP controls lysosomal transport by inducing the recruitment of dynein-dynactin motors. Curr Biol 2001; 11: 1680-1685. 
33. Vieira OV, Bucci C, Harrison RE, Trimble WS, Lanzetti L, Gruenberg J et al. Modulation of Rab5 and Rab7 recruitment to phagosomes by phosphatidylinositol 3-kinase. Mol Cell Biol 2003; 23: 2501-2514.

34. Horiuchi H, Lippe R, McBride HM, Rubino M, Woodman P, Stenmark H et al. A novel Rab5 GDP/GTP exchange factor complexed to Rabaptin-5 links nucleotide exchange to effector recruitment and function. Cell 1997; 90: 1149-1159.

35. Blumer J, Rey J, Dehmelt L, Mazel T, Wu YW, Bastiaens P et al. RabGEFs are a major determinant for specific Rab membrane targeting. J Cell Biol 2013; 200: 287-300.

36. Haglund K, Dikic I. The role of ubiquitylation in receptor endocytosis and endosomal sorting. J Cell Sci 2012; 125(Part 2): 265-275.

37. Kim PK, Hailey DW, Mullen RT, Lippincott-Schwartz J. Ubiquitin signals autophagic degradation of cytosolic proteins and peroxisomes. Proc Natl Acad Sci USA 2008; 105: 20567-20574.

38. Mattera R, Bonifacino JS. Ubiquitin binding and conjugation regulate the recruitment of Rabex-5 to early endosomes. EMBO J 2008; 27: 2484-2494.

39. Penengo L, Mapelli M, Murachelli AG, Confalonieri S, Magri L, Musacchio A et al. Crystal structure of the ubiquitin binding domains of rabex-5 reveals two modes of interaction with ubiquitin. Cell 2006; 124: 1183-1195.

40. Dantuma NP, Groothuis TA, Salomons FA, Neefjes J. A dynamic ubiquitin equilibrium couples proteasomal activity to chromatin remodeling. J Cell Biol 2006; 173: 19-26.

41. Sims JJ, Scavone F, Cooper EM, Kane LA, Youle RJ, Boeke JD et al. Polyubiquitin-sensor proteins reveal localization and linkage-type dependence of cellular ubiquitin signaling. Nat Methods 2012; 9: 303-309.

42. Eckelman BP, Salvesen GS, Scott FL. Human inhibitor of apoptosis proteins: why XIAP is the black sheep of the family. EMBO Rep 2006; 7: 988-994.

43. Schile AJ, Garcia-Fernandez M, Steller H. Regulation of apoptosis by XIAP ubiquitin-ligase activity. Genes Dev 2008; 22: 2256-2266.

44. Flanagan L, Sebastia J, Tuffy LP, Spring A, Lichawska A, Devocelle M et al. XIAP impairs Smac release from the mitochondria during apoptosis. Cell Death Dis 2010; 1: e49.

45. Kushnareva Y, Newmeyer DD. Bioenergetics and cell death. Ann NY Acad Sci 2010; 1201: 50-57.

46. Alexandrov K, Horiuchi H, Steele-Mortimer O, Seabra MC, Zerial M. Rab escort protein-1 is a multifunctional protein that accompanies newly prenylated rab proteins to their target membranes. EMBO J 1994; 13: 5262-5273.

47. Sarraf SA, Raman M, Guarani-Pereira V, Sowa ME, Huttlin EL, Gygi SP et al. Landscape of the PARKIN-dependent ubiquitylome in response to mitochondrial depolarization. Nature 2013; 496: 372-376.

48. Cesari R, Martin ES, Calin GA, Pentimalli F, Bichi R, McAdams $\mathrm{H}$ et al. Parkin, a gene implicated in autosomal recessive juvenile parkinsonism, is a candidate tumor suppressor gene on chromosome 6q25-q27. Proc Natl Acad Sci USA 2003; 100 5956-5961.

49. Johnson BN, Berger AK, Cortese GP, Lavoie MJ. The ubiquitin E3 ligase parkin regulates the proapoptotic function of Bax. Proc Natl Acad Sci USA 2012; 109: 6283-6288.

50. Nakatani Y, Kleffmann T, Linke K, Condon SM, Hinds MG, Day CL. Regulation of ubiquitin transfer by XIAP, a dimeric RING E3 ligase. Biochem J 2013; 450: 629-638.

51. Liu Z, Sun C, Olejniczak ET, Meadows RP, Betz SF, Oost T et al. Structural basis for binding of Smac/DIABLO to the XIAP BIR3 domain. Nature 2000; 408 : 1004-1008.

52. Silke J, Ekert PG, Day CL, Hawkins CJ, Baca M, Chew J et al. Direct inhibition of caspase 3 is dispensable for the anti-apoptotic activity of XIAP. EMBO J 2001; 20: 3114-3123.

53. Morizane Y, Honda R, Fukami K, Yasuda H. X-linked inhibitor of apoptosis functions as ubiquitin ligase toward mature caspase-9 and cytosolic Smac/DIABLO. J Biochem 2005; 137: 125-132.

54. Salvesen GS, Duckett CS. IAP proteins: blocking the road to death's door. Nat Rev Mol Cell Biol 2002; 3: 401-410.

55. MacFarlane M, Merrison W, Bratton SB, Cohen GM. Proteasome-mediated degradation of Smac during apoptosis: XIAP promotes Smac ubiquitination in vitro. J Biol Chem 2002; 277: 36611-36616.

56. Owens TW, Foster FM, Valentijn A, Gilmore AP, Streuli CH. Role for X-linked inhibitor of apoptosis protein upstream of mitochondrial permeabilization. J Biol Chem 2010; 285 1081-1088.

57. Weiss JN, Korge $\mathrm{P}$, Honda HM, Ping P. Role of the mitochondrial permeability transition in myocardial disease. Circ Res 2003; 93: 292-301.

58. Duchen MR, Leyssens A, Crompton M. Transient mitochondrial depolarizations reflect focal sarcoplasmic reticular calcium release in single rat cardiomyocytes. J Cell Biol 1998; 142: $975-988$.

59. Brady NR, Elmore SP, van Beek JJ, Krab K, Courtoy PJ, Hue L et al. Coordinated behavior of mitochondria in both space and time: a reactive oxygen species-activated wave of mitochondrial depolarization. Biophys J 2004; 87: 2022-2034.

60. Ghosh P, Griffith J, Geuze HJ, Kornfeld S. Mammalian GGAs act together to sort mannose 6-phosphate receptors. J Cell Biol 2003; 163: 755-766.

61. Pan X, Rudolph JM, Abraham L, Habermann A, Haller C, Krijnse-Locker J et al. HIV-1 Nef compensates for disorganization of the immunological synapse by inducing trans-Golgi network-associated Lck signaling. Blood 2012; 119: 786-797.

62. Slot JW, Geuze HJ, Gigengack S, Lienhard GE, James DE. Immuno-localization of the insulin regulatable glucose transporter in brown adipose tissue of the rat. J Cell Biol 1991; 113: $123-135$.

\section{Supplementary Information accompanies this paper on Cell Death and Differentiation website (http://www.nature.com/cdd)}

